ARTICLE

Received 5 Aug 2015 | Accepted 24 May 2016 | Published 4 Jul $2016 \quad$ DOl: 10.1038/ncomms12044 OPEN

\title{
COPS5 amplification and overexpression confers tamoxifen-resistance in ER $\alpha$-positive breast cancer by degradation of NCoR
}

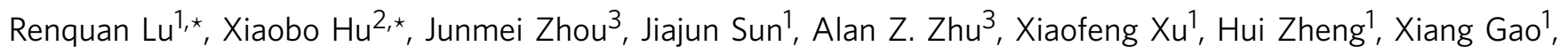
Xian Wang ${ }^{4}$, Hongchuan $\mathrm{Jin}^{4}$, Ping $\mathrm{Zhu}^{3} \&$ Lin Guo ${ }^{1}$

Oestrogen receptor $\alpha(E R \alpha)$ antagonists are used in endocrine therapies for $E R \alpha$-positive $(E R \alpha+)$ breast cancer patients. Unfortunately the clinical benefit is limited due to intrinsic and acquired drug resistance. Here using integrated genomic and functional studies, we report that amplification and/or overexpression of COPS5 (CSN5/JAB1) confers resistance to tamoxifen. Amplification and overexpression of COPS5, a catalytic subunit of the COP9 complex, is present in about $9 \%$ of the ER $\alpha+$ primary breast cancer and more frequently $(86.7 \%, 26 / 30)$ in tamoxifen-refractory tumours. Overexpression of COPS5, through its isopeptidase activity, leads to ubiquitination and proteasome-mediated degradation of $\mathrm{NCOR}$, a key corepressor for ER $\alpha$ and tamoxifen-mediated suppression of ER $\alpha$ target genes. Importantly, COPS5 overexpression causes tamoxifen-resistance in preclinical breast cancer models in vitro and in vivo. We also demonstrate that genetic inhibition of the isopeptidase activity of COPS5 is sufficient to re-sensitize the resistant breast cancer cells to tamoxifen-treatment, offering a potential therapeutic approach for endocrine-resistant breast cancer patients.

\footnotetext{
${ }^{1}$ Department of Clinical Laboratory, Shanghai Cancer Center, Fudan University; Department of Oncology, Shanghai Medical School, Fudan University, Shanghai 200032, China. ${ }^{2}$ Department of Laboratory Medicine, Long Hua Hospital, Shanghai University of TCM, Shanghai 200032 , China. ${ }^{3}$ Gerchi Biotech Research Laboratories, 100 Research Park, Zhejiang 314300, China. ${ }^{4}$ Department of Medical Oncology, Sir Run Run Shaw Hospital, Zhejiang 310016, China. ${ }^{\star}$ These authors contributed equally to this work. Correspondence and requests for materials should be addressed to P.Z. (email: pizhu_sh@163.com) or to L.G. (email: guolin500@hotmail.com).
} 
$\mathrm{B}$ reast cancer is the most common cancer in women worldwide, with nearly 1.7 million new cases diagnosed each year. WHO (World Health Organization) estimated that worldwide over 521,000 women died in 2012 due to breast cancer. About $80 \%$ of the breast cancer patients express oestrogen receptor $\alpha(\mathrm{ER} \alpha)$ in their tumours, which is essential for normal mammalian gland development and breast cancer development and progression. Therefore, ER $\alpha$ antagonists, for example, tamoxifen and fulvestrant, and aromatase inhibitors (AIs) that block the ER signalling are widely used in endocrine therapies for $\mathrm{ER} \alpha$-positive $(\mathrm{ER} \alpha+)$ breast cancer patients ${ }^{1-3}$. Tamoxifen is the most prescribed ER antagonist particularly in the first-line therapy. Unfortunately the clinical benefit is limited because of intrinsic and acquired drug resistance. The appearance and development of resistant tumours are major obstacles in controlling the growth and metastasis of breast cancer. A number of mechanisms have been associated with resistance to tamoxifen ${ }^{4,5}$. Activation of the receptor tyrosine kinases, particularly ERBB2/HER2, EGFR and IGF1R, and other kinase pathways, for example, PI3K/AKT/mTOR, have been shown to contribute to endocrine-resistance ${ }^{6-8}$. Apoptosis and cell cycle regulators appear to play roles in refractory to endocrine therapy in breast cancer as well ${ }^{8,9}$. Dysregulation of $\mathrm{ER} \alpha$ coregulators, including coactivators p160s/NCoAs and corepressors NCoR and SMRT, have been shown to be crucial in switching antagonistic selective ER modulators (SERMs) to agonists $^{10,11}$. In addition, hotspot mutations in the ligandbinding domain of $\mathrm{ER} \alpha$, which confer partial resistance to current endocrine therapies including AIs, SERMs and selective ER degraders (SERDs), were observed in $\sim 20 \%$ of the endocrine-refractory metastatic breast cancer ${ }^{12-16}$. Activation of the kinase and anti-apoptotic pathways, loss of ER $\alpha$ expression by genetic or epigenetic mechanisms, stem cells or epithelialmesenchymal transition (EMT), and autophagocytosis can also account for $E R \alpha$-independent resistance to endocrine therapies $^{4,17}$. Together, it appears that breast cancer can utilize multiple molecular approaches to development of endocrine therapy refractory. Uncovering novel mechanisms that are prevalent in resistant tumours is of importance to develop new therapeutic strategies for drug-resistant patients.

Here using integrated genomic and functional studies, we report that amplification and/or overexpression of the COP9 complex-associated isopeptidase COPS5 is presented in about $9 \%$ of the $\mathrm{ER} \alpha+$ primary breast cancer and more frequently $(86.7 \%, 26 / 30)$ in tamoxifen-resistant tumours. Mechanistically, amplification and overexpression of COPS5 lead to an ubiquitination/proteasome-dependent destabilization of NCoR protein, a corepressor required for tamoxifen-mediated suppression of $\mathrm{ER} \alpha$ target genes, which is considered as a key mechanism of action of the drug. In turn, COPS5 overexpression confers resistance to tamoxifen by converting it to a potent $\mathrm{ER} \alpha$ agonist. We also show evidence that inhibition of the isopeptidase activity of COPS5 is sufficient to re-sensitize the resistant breast cancer cells to tamoxifen-treatment, providing a potential therapeutic approach for endocrine-resistant breast cancer patients.

\section{Results}

COPS5 overexpression in tamoxifen-resistant breast cancer. To explore the clinically relevant mechanisms associated with tamoxifen-resistance, we analysed both The Cancer Genome Atlas (TCGA) breast cancer data, which contains mainly primary and untreated or lightly treated patient samples, and our internal breast patient samples collected pre- and post-tamoxifentreatment. COPS5 is one of the frequently altered genes in
$\mathrm{ER} \alpha+$ breast cancer samples from TCGA. COPS5 gene is located at the chromosome $8 \mathrm{q} 13$ which is amplified (copy number $(\mathrm{CN})>6)$ in about $9 \%(70 / 774)$ of the $\mathrm{ER} \alpha+$ breast cancer (Log2 ESR1 expression > 5). Although in general the amplicon is relatively broad and not scored as a significant GISTIC peak in a recent pan- or lineage-specific TCGA cancer analysis ${ }^{18,19}$, there is a trend of COPS5-centric focality, particularly in the patients with high copy number of COPS5 and focal amplification (Fig. 1a). Importantly, the expression of COPS5 mRNA correlates with the $\mathrm{CN}\left(R^{2}=0.61\right.$, Pearson's $\chi^{2}$-test $\left.P<10^{-15}\right)$, suggesting a potential functional relevance of COPS5 amplification (Fig. 1b). Of note, high levels of amplitude $(\mathrm{CN}>12)$ of COPS5 are significantly enriched in the $\mathrm{ER} \alpha+$ breast cancer patients, implicating that the amplification and overexpression of COPS5 may contribute to the ER $\alpha$-related signalling and tumour progression (Fig. 1c, Supplementary Table 1). In addition, the clinical outcomes (disease-free survival rate and overall survival rate) of the TCGA breast cancer patients (including luminal $\mathrm{A}$ and $\mathrm{B}$ ) with high expression levels of COPS5 appeared to be poorer (Supplementary Fig. 1a,b). In addition, the worse clinical outcomes are unlikely to be associated with tumour subtypes because COPS5 expression was similar in luminal A and B tumours (Supplementary Fig. 1c). To evaluate if COPS5 protein overexpression is involved in tamoxifen-resistance, we investigated $\mathrm{ER} \alpha+$ breast tumours that were untreated $(n=31)$ or refractory to tamoxifen-treatment $(n=30)$. Notably, COPS5 is mainly expressed in cellular nucleus of cancer cells (Fig. 1d). Based on the immunohistochemistry (IHC) staining scores (refer to Methods), the rate of high expression ( + to +++ ) of COPS5 protein is significantly elevated in tamoxifenrefractory breast tumours $(86.7 \%, 26 / 30)$ compared with untreated breast tumours $(25.8 \%, 8 / 31)$ (Fig. 1d,e, Supplementary Table 2). To further evaluate the role of COPS5 in a model system, we attempted to establish in vitro models derived from MCF7 $\mathrm{ER} \alpha+$ breast cancer cells that are highly resistant to 4-hydroxy-tamoxifen (4OHT, an active metabolite of tamoxifen) at up to $2 \mu \mathrm{M}$ by a dose-escalation protocol (refer to Methods) (Fig. 1f). These clones are also partially resistant to the SERD fulvestrant but not the pan-cytotoxic CDK inhibitor flavopiridol (Supplementary Fig. 2), suggesting that resistance mechanism is specific to antiestrogens. Strikingly, among five resistant clones, we identified two clones with amplified $\mathrm{CN}$ of COPS5 (Fig. 1g), and increased mRNA (Fig. 1h) and protein expression levels of COPS5 (Fig. 1i). Furthermore, 4OHT showed potent agonistic instead of antagonistic or weak agonistic activity in both clones as tested by the expression of six ER $\alpha$ target genes (Fig. 1j, Supplementary Fig. 3), suggesting a general role of COPS5 overexpression in activation of ER $\alpha$-dependent transcription in response to 4OHT. Therefore, the clones \#1 and \#3 recapitulated COPS5 overexpression observed in clinical tamoxifen-refractory tumours, and were used for further studies of the mechanisms of tamoxifen-resistance. In summary, in both clinical breast tumour samples and in vitro cell line models we observed that amplification and overexpression of COPS5 was associated with endocrine/tamoxifen-resistance.

Overexpression of COPS5 confers resistance to tamoxifen. To test if overexpression of COPS5 plays a causative role in driving resistance to tamoxifen, we next established MCF7 cell lines stably expressing wild-type (WT) and metalloprotease-deficient D151N COPS5 (refs 20,21) by lentiviral infection (Fig. 2a). Of note, ER $\alpha$ expression was not altered by either WT or D151N COPS5 expression (Fig. 2a). In the absence of additional estradiol in culture medium containing full foetal bovine serum (FBS), overexpression of COPS5 did not change the basal expression of 
a

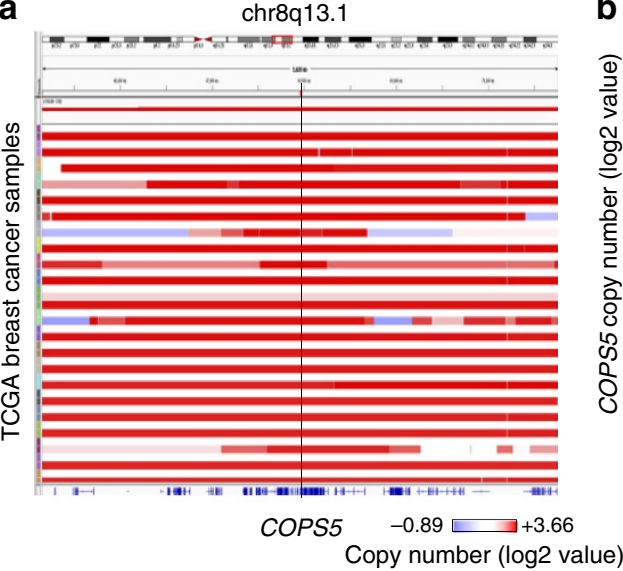

b

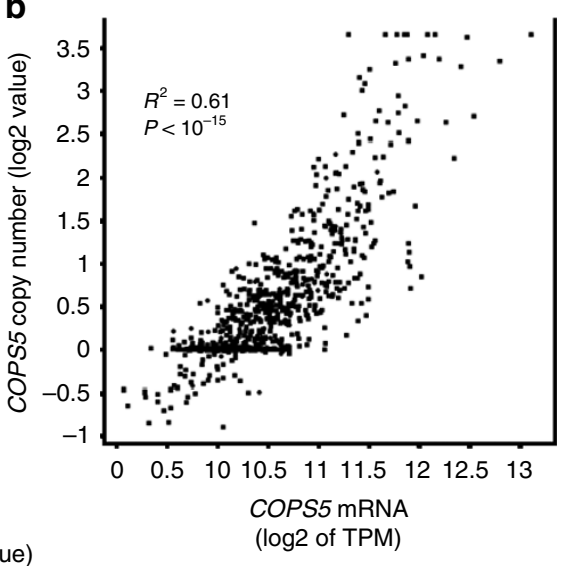

C

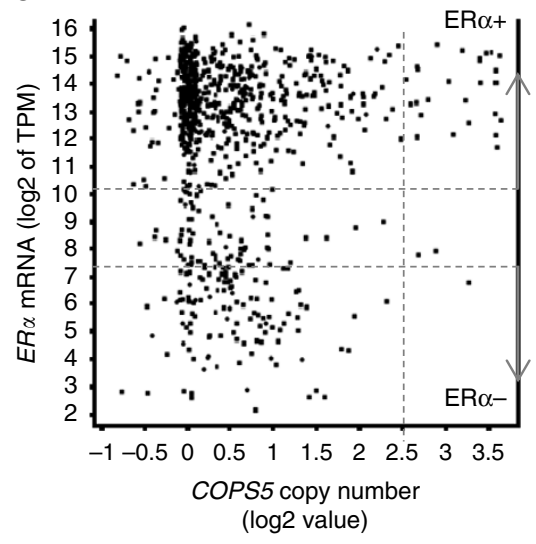

d
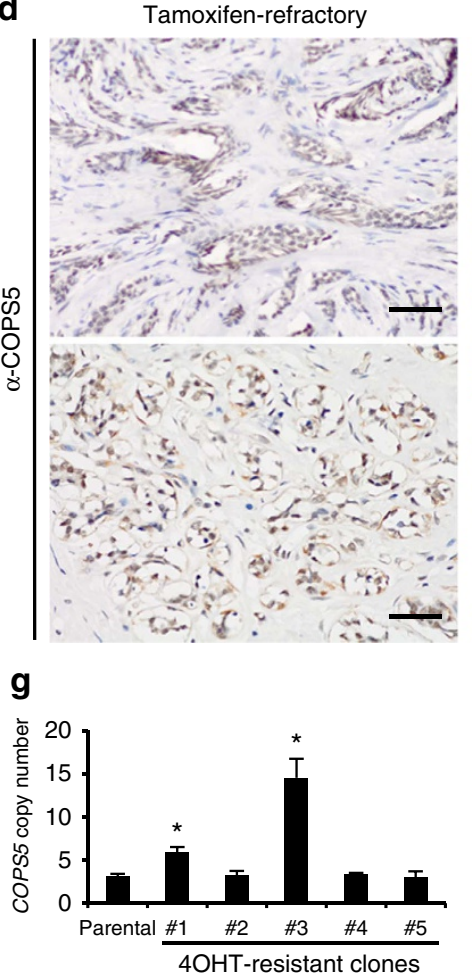

Tamoxifen-untreated

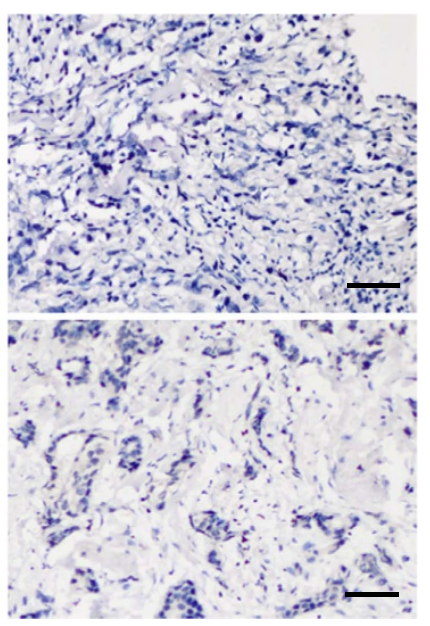

h

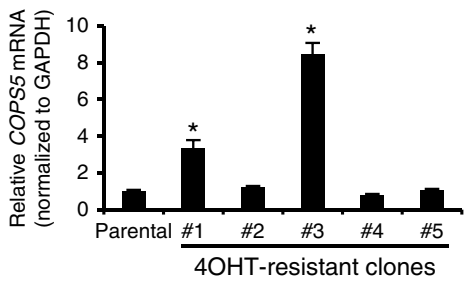

e

f

\begin{tabular}{|c|c|c|c|c|}
\hline \multirow{2}{*}{ Group } & \multirow{2}{*}{ Number } & \multicolumn{2}{|c|}{ COPS5 score } & \multirow{2}{*}{\begin{tabular}{c}
$P$ \\
\cline { 3 - 4 }
\end{tabular}} \\
\cline { 3 - 4 } & & $+\sim+++$ & - & value \\
\hline TAM-refractory & 30 & 26 & 4 & \multirow{2}{*}{$<10^{-5}$} \\
\hline TAM-untreated & 31 & 8 & 23 & \\
\hline
\end{tabular}

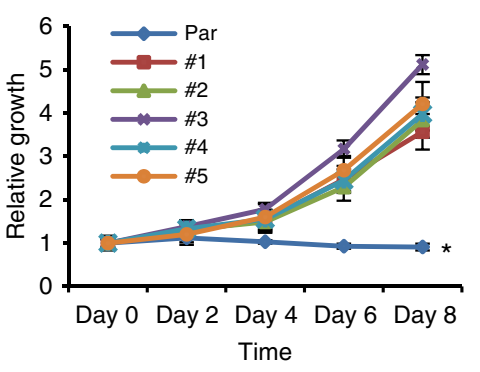

i

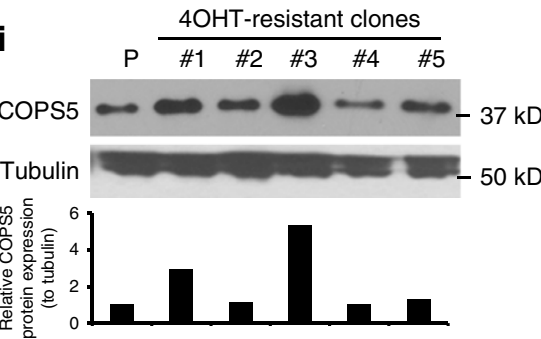

j
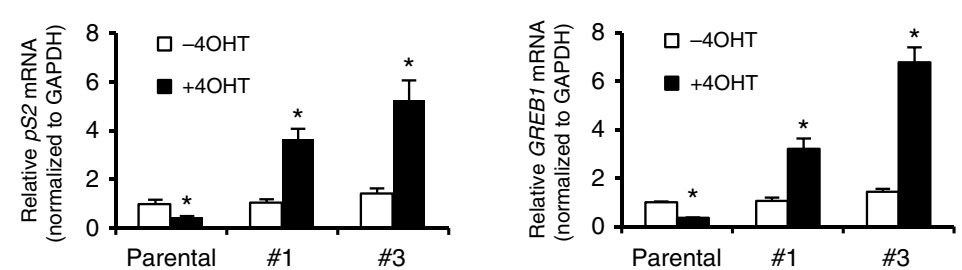

Figure 1 | COPS5 is amplified and overexpressed in ER $\boldsymbol{\alpha}+$ breast cancer and tamoxifen-resistant MCF7 cells. (a) Focal amplification of COPS5 in TCGA breast cancer. Samples were sorted by COPS5 amplitude from the IGV of TCGA portal developed by the Broad Institute. (b) Correlation of mRNA level and copy number of COPS5 in TCGA breast cancer samples. (c) COPS5 copy number and ER $\alpha$ mRNA expression in TCGA breast cancer samples. (d) Immunohistochemistry of COPS5 in representative tamoxifen-untreated and refractory breast cancer. Anti-COPS5 antibody showed positive staining in the nucleus of tumour cells (scale bar, $50 \mu \mathrm{m}$ ). (e) $\chi^{2}$-calculation of the significance of COPS5-overexpression rates in tamoxifen-refractory and tamoxifenuntreated breast tumours. (f) Growth curve of parental and tamoxifen-resistant MCF7 clones established by in vitro dose-escalation 4OHT-treatment (refer to Methods) in the presence of $2 \mu \mathrm{M}$ of $4 \mathrm{OHT}$. Values are normalized to day 0 treatment. ${ }^{*}$ Significant difference compared with other samples $(P<0.01$ at both day 6 and day 8, Student's t-test). (g) Copy number of COPS5 genomic DNA normalized to LINE1 in parental and tamoxifen-resistant MCF7 clones. *Significant difference compared with parental MCF7 ( $P<0.01$, Student's t-test). (h) Expression of COPS5 mRNA in parental and tamoxifen-resistant MCF7 clones. Data were normalized to GAPDH and relative to COPS5 expression in the parental cell line which was set as 1 . *Significant difference compared with parental MCF7 ( $P<0.01$, Student's t-test). (i) Western blot analysis and quantification of COPS5 protein expression in parental and tamoxifen-resistant MCF7 clones. (j) Quantitative RT-PCR analysis of ER $\alpha$ target genes pS2 and GREB1 in MCF7 clones after treatment with $500 \mathrm{nM} 4 \mathrm{OHT}$ for $48 \mathrm{~h}$. Data are presented as mean + s.e.m. of three biological replicates. ${ }^{\star}$ Significant difference compared with untreated parental MCF7 $(P<0.01$, Student's $t$-test). 

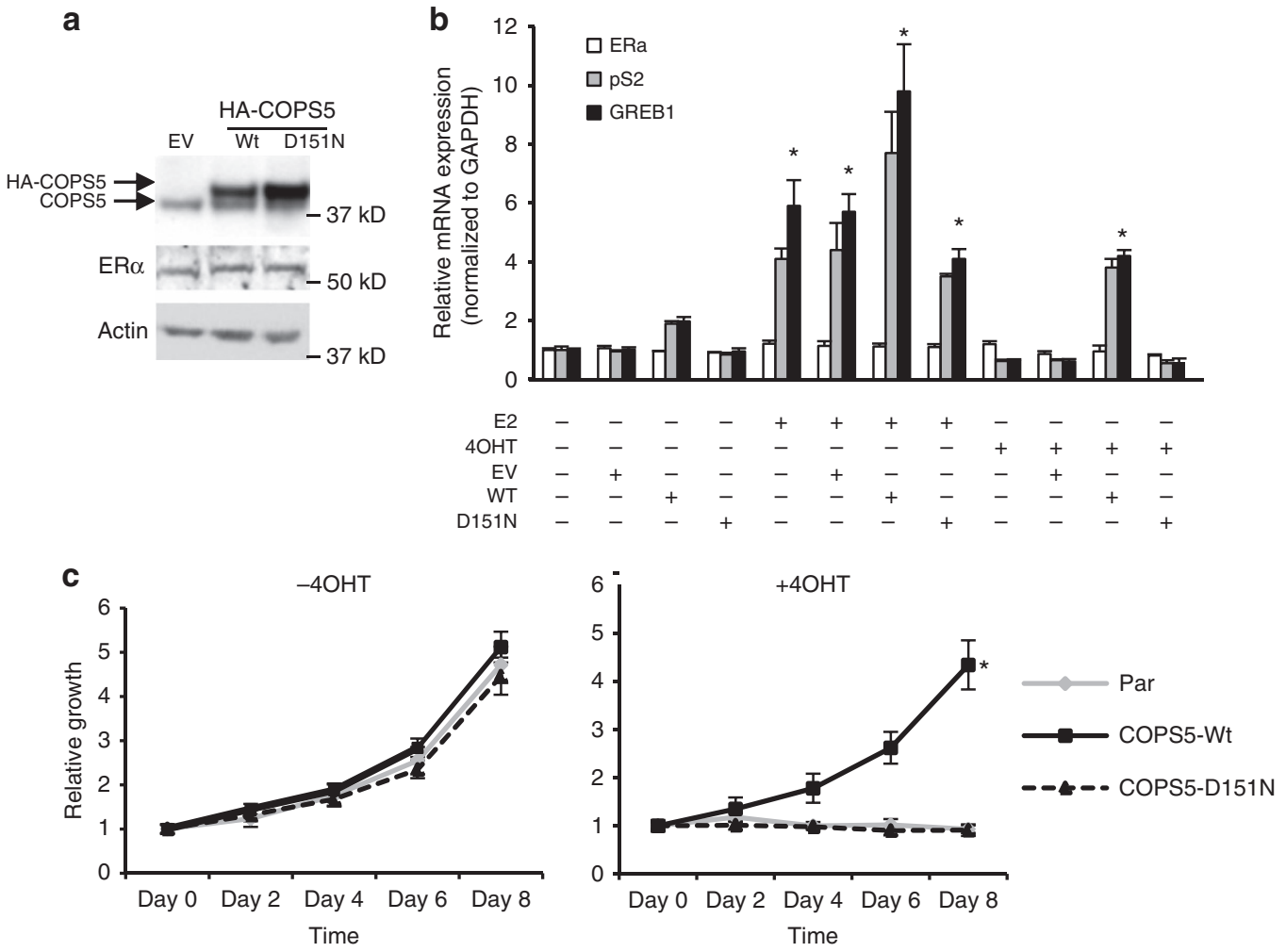

Figure 2 | Overexpression of wild-type COPS5 confers resistance to 40HT. (a) Western blot analysis of COPS5 expression in MCF7 cell lines stably engineered with empty vector (EV), WT or D151N COPS5 CDNA. (b) Quantitative RT-PCR analysis of ER $\alpha$ and its target genes $p S 2$ and GREB1 by ER $\alpha$ ligand treatment ( $48 \mathrm{~h}$ ) in MCF7 cell lines stably engineered with empty vector (EV), WT or D151N COPS5 CDNA. Data are presented as mean + s.e.m. of three biological replicates. E2: $1 \mathrm{nM}, 4 \mathrm{OHT}$ : $500 \mathrm{nM}$. *Significant difference compared with basal (untreated) levels $(P<0.01$, Student's t-test). (c) Growth of MCF7 cell lines stably engineered with empty vector (Par), WT or D151N COPS5 cDNA in the absence or presence of $40 \mathrm{OHT}(1 \mu \mathrm{M})$. Data are presented as mean + s.e.m. of three biological replicates. ${ }^{\star}$ Significant difference compared with other samples $(P<0.01$, Student's $t$-test $)$.

$\mathrm{ER} \alpha$ target genes $p S 2$ (TFF1) and GREB1 (Fig. 2b, left four panels). With addition of E2, the expression of both target genes was induced by four to sixfold, while WT COPS5 only marginally induced expression of these genes (Fig. 2b, middle four panels). In the presence of 4OHT, while both $p S 2$ and GREB1 were suppressed in parental, vector control and D151N COPS5-overexpressing cell lines, overexpression of WT COPS5 significantly increased the target gene expression (Fig. $2 \mathrm{~b}$, right four panels, Student's $t$-test $P<0.01$ ), indicating that overexpression of COPS5, depending on its catalytic activity, can convert the antagonistic $4 \mathrm{OHT}$ to an $\mathrm{ER} \alpha$ agonist in breast cancer cells, a hallmark feature of endocrine-resistance at molecular level ${ }^{11}$. Interestingly, the mRNA expression of $\mathrm{ER} \alpha$ was not altered by COPS5, suggesting that regulation of ER $\alpha$ target gene expression is likely through a coregulator-related mechanism (Fig. 2b, white bars). In addition to $p S 2$ and GREB1, we examined five more known ER $\alpha$ target genes: PGR which is activated by E2 but inhibited by $4 \mathrm{OHT}$ in parental MCF7; NRIP, IGFBP4, CTSD for which E2 acts as an agonist while $4 \mathrm{OHT}$ acts as a weak agonist as well, and BMP7 for which E2 is an antagonist. Interestingly, $4 \mathrm{OHT}$ strongly induced all target genes except $B M P 7$ by overexpression of COPS5-WT, but not D151N (Supplementary Fig. 4). These data support the generality of the role of COPS5 amplification/overexpression in switching $4 \mathrm{OHT}$ from a SERM to a pure agonist. We further investigated the cell growth phenotype of these MCF7 cell lines. In the absence of 4OHT, WT or D151N COPS5-overexpressing cells did not demonstrate any difference in growth rate in vitro compared with the parental cells. However, WT COPS5 cells were still able to proliferate on treatment with 4OHT, whereas parental and D151N-overexpressed cells were inhibited by the antagonist (Fig. 2c). Similarly, in another ER $\alpha+$ breast cancer cell line T47D, we observed that overexpression of WT but not D151N COPS5 led to agonistic conversion of $4 \mathrm{OHT}$ in ER $\alpha$-dependent transcription (Supplementary Fig. 5a,b), and caused resistance to 4OHT-induced growth inhibition (Supplementary Fig. 5c). Together, these data support that amplification and overexpression of COPS5 in $\mathrm{ER} \alpha+$ breast cancer cells confers resistance to tamoxifen.

COP9 complex induces tamoxifen-resistance by degrading NCoR. Next we sought to elucidate the mechanisms underlying COPS5-mediated tamoxifen-resistance. The $\mathrm{Zn}^{2+}$-dependent isopeptidase/deneddylase COPS5 is the catalytic component of the COP9 signalosome complex which is involved in regulating the activity of Skp, Cullin, F-box containing (SCF) E3 ubiquitin ligases and subsequently the stability of substrates ${ }^{22,23}$. To explore the potential targets of COPS5 in ER $\alpha$ regulated transcription, we performed co-immunoprecipitation experiments in tamoxifen-sensitive parental MCF7 cells. We confirmed that COPS5 co-existed with other COP9 components, for example, GPS1/CSN1 and CSN2 (Fig. 3a). Intriguingly, while $\mathrm{ER} \alpha$, coactivator PCAF and corepressor HDAC1 did not appear to associate with COPS5/COP9, the NCoR corepressor complex, including NCoR and HDAC3, was effectively pulled down with the COP9 subunits (Fig. 3a). Reciprocally, immuneprecipitation of endogenous NCoR also pulled down COPS5 (Supplementary Fig. 6a). Indeed, we observed a remarkable reduction of $\mathrm{NCoR}$ protein expression in the tamoxifen-resistant MCF7 clones (\#1 and \#3) in which COPS5 is overexpressed (Figs 1i and 3b), 
a

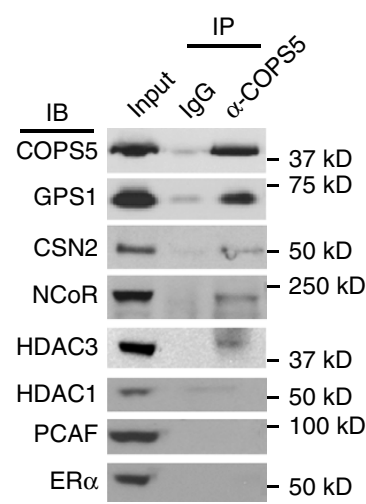

b

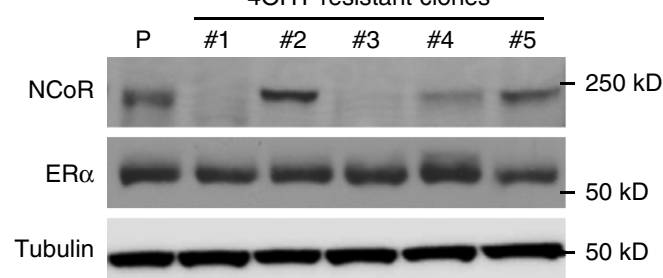

d

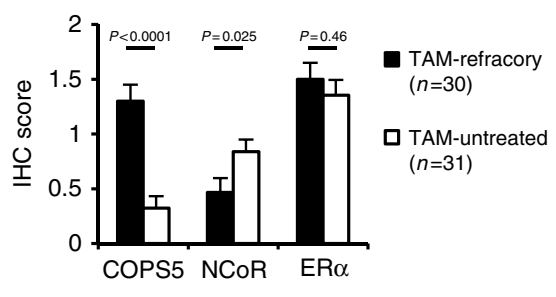

C
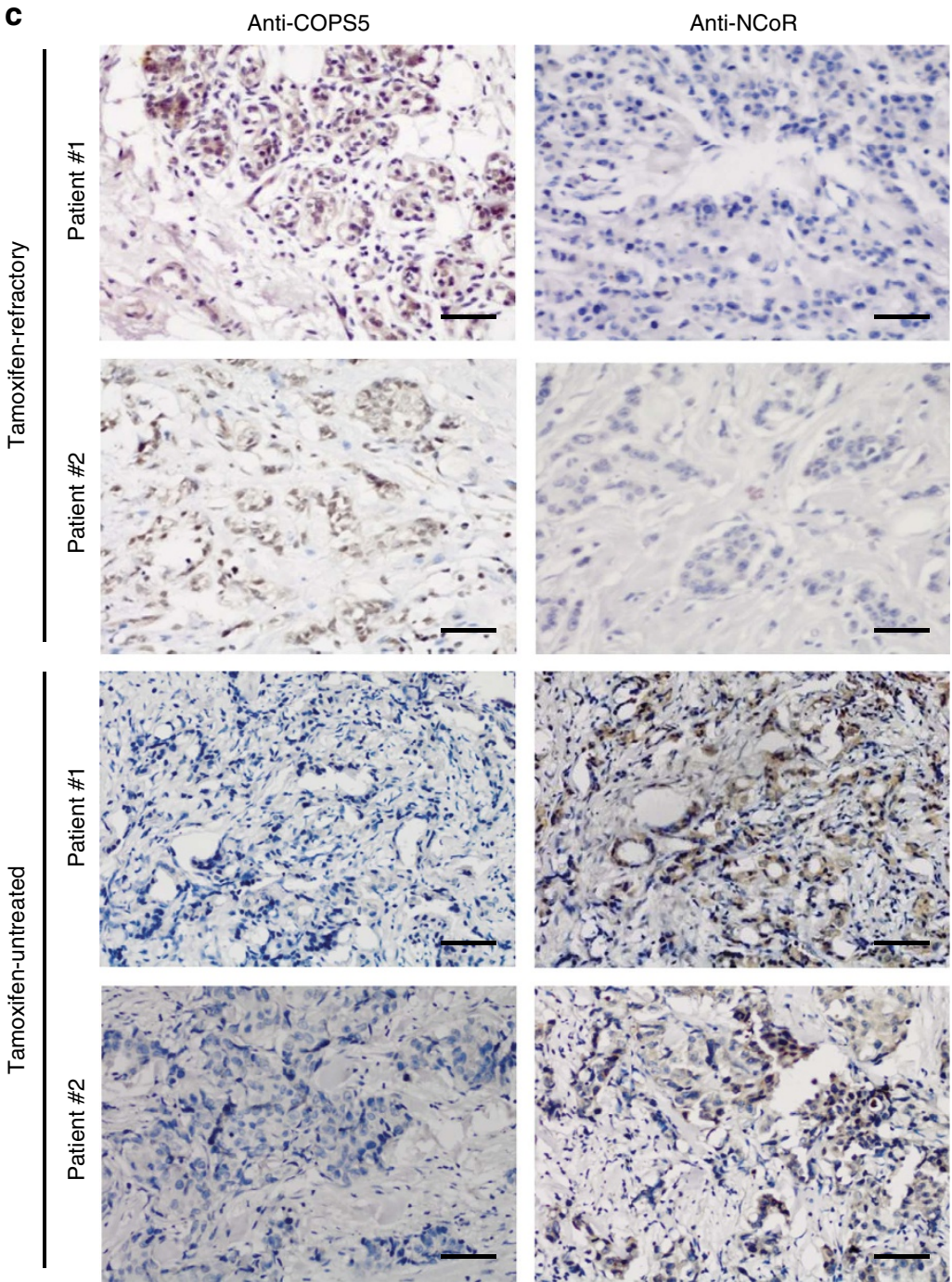
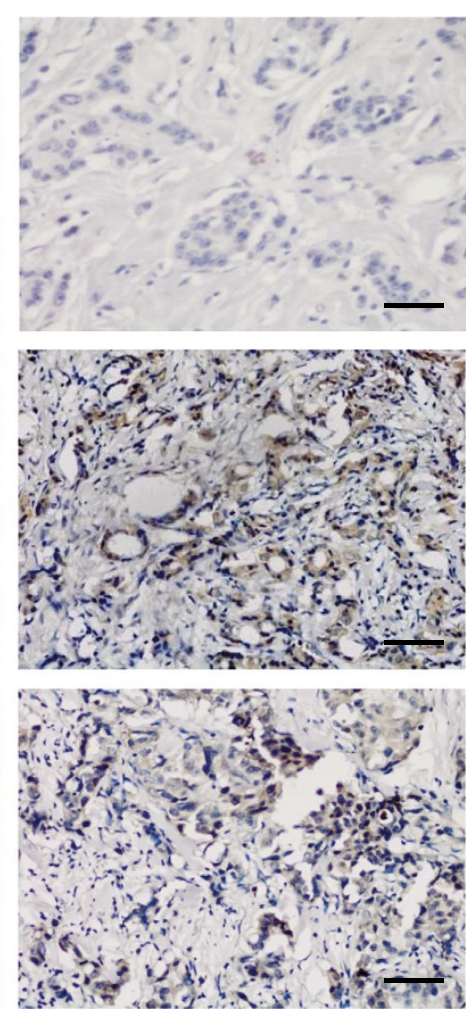

Figure 3 | NCoR protein is downregulated in COPS5-overexpressed breast cancer cells. (a) Western blot analysis of proteins that were coimmunoprecipitated with anti-COPS5 antibodies in MCF7 parental cells. (b) Western blot analysis of NCoR and ER $\alpha$ expression in parental and tamoxifen-resistant MCF7 clones. P, parental cell line. (c) IHC of NCoR and COPS5 expression in representative tamoxifen-untreated and refractory breast cancer samples (scale bar, $50 \mu \mathrm{m}$ ). Anti-COPS5 and anti-NCoR antibodies showed nuclear and nuclear/cytoplasmic staining in tumor cells, respectively. (d) Quantification and statistical analysis of IHC staining of COPS5, NCoR and ER $\alpha$ in tamoxifen-untreated and refractory breast cancer samples.

suggesting a functional interaction between COP9 and NCoR complex. Of note, ER $\alpha$ has been proposed to be destabilized by COP9/COPS5 in an agonist-dependent manner ${ }^{24}$. However, in the tamoxifen-resistance MCF7 cell lines, we did not detect genomic alterations of the ESR 1 gene which encodes $\mathrm{ER} \alpha$, and $\mathrm{ER} \alpha$ protein was expressed at similar levels in these clones compared with parental MCF7 cells (Fig. 3b). We also investigated tamoxifen-naive $(n=31)$ and refractory $(n=30)$ breast tumour samples used in Fig. 1, and observed an inverse correlation between NCoR and COPS5 protein levels by IHC staining (Fig. 3c). We then quantified the IHC staining for COPS5, NCoR and ER $\alpha$ (Supplementary Table 2). Intriguingly, while COPS5 expressed significantly higher in refractory tumours (Pearson's $\chi^{2}$-test $P<0.0001$ ), it is also significant that $\mathrm{NCoR}$ expression is decreased in tamoxifen-resistant breast tumours (Pearson's $\chi^{2}$-test $P=0.025$ ) (Fig. 3d). In contrast, $\mathrm{ER} \alpha$ expression showed no significant difference between tamoxifen-naive and refractory groups (Pearson's $\chi^{2}$-test $P=0.46)$, suggesting that $\mathrm{ER} \alpha$ expression is not a key downstream event of COPS5 overexpression (Fig. 3d, Supplementary Table 2).
We then hypothesized that COPS5 regulated the protein stability of NCoR through COP9-mediated regulation ubiquitination/proteasomal activity. First we performed a cycloheximide (CHX) chase assay to measure the half-life of NCoR in the absence and presence of COPS5 overexpression (Fig. 4a). While half-life of NCoR protein is $\sim 3.1 \mathrm{~h}$ in parental MCF7 cells, overexpression of COPS5-WT significantly reduced the half-life to $0.34 \mathrm{~h}$ (Fig. $4 \mathrm{a}, \mathrm{b}$ ). In contrast, overexpression of the isopeptidase-deficient COPS5-D151N did not alter the half-life of NCoR (3.7 h) substantially (Fig. 4a,b), suggesting the importance of the catalytic activity. Next, we noticed that NCoR protein, rather than mRNA, was downregulated by overexpression of WT COPS5 in parental MCF7 cells (Fig. 4c, Supplementary Fig. 6b). In the presence of proteasome inhibitor MG132, NCoR protein expression was restored to basal level (Fig. 4c). Again, the $\mathrm{ER} \alpha$ protein level was not changed in these cells (Fig. 4c). We then conducted in vivo ubiquitination assays by which we demonstrated that overexpression of WT COPS5 augmented ubiquitination of NCoR while the D151N mutant partially antagonized NCoR ubiquitination (Fig. 4d), presumably due to the dominant negative function ${ }^{20}$. Interestingly, both K63R and 


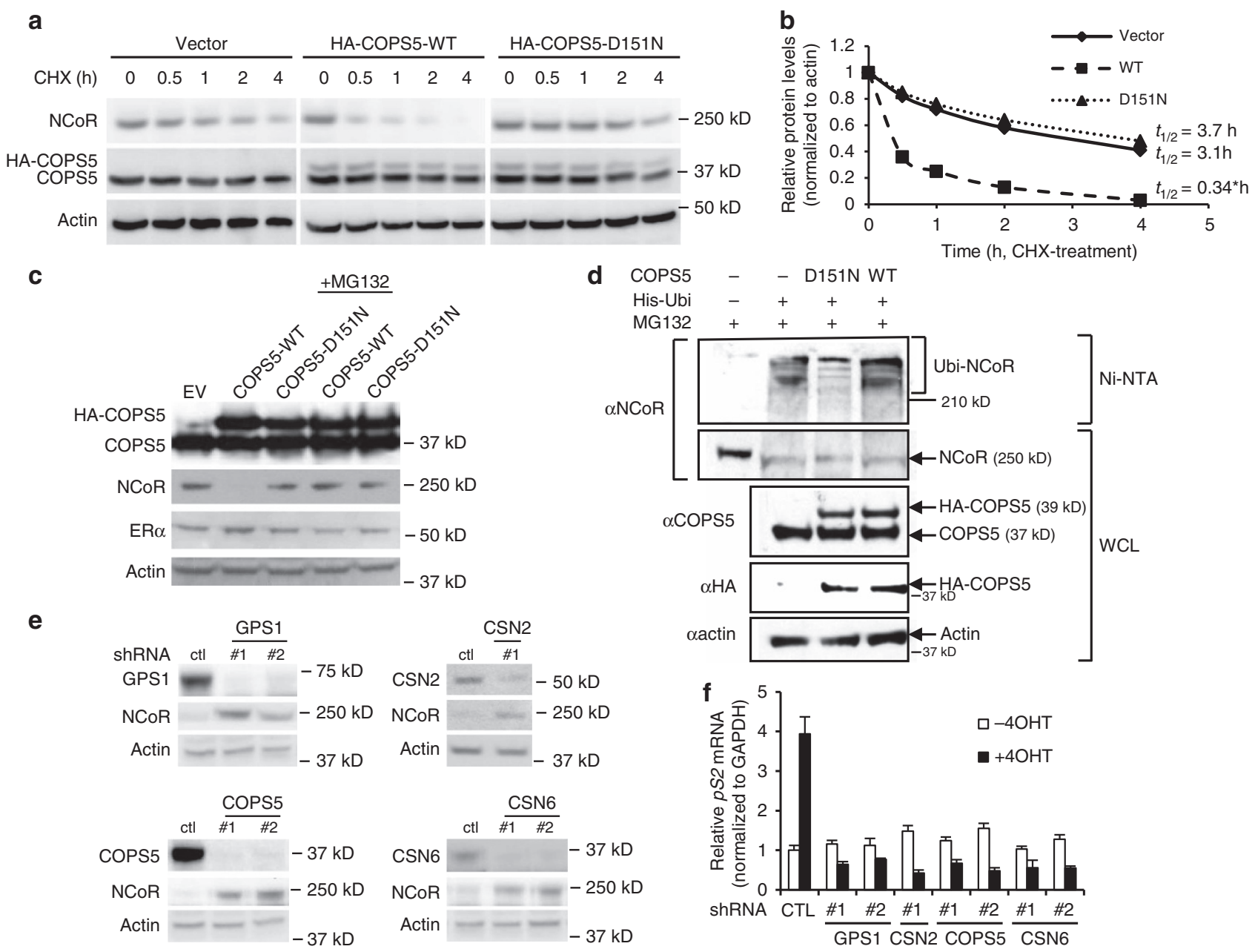

Figure 4 | COPS5 overexpression leads to degradation of NCoR protein through the ubiquitin-proteasome pathway. (a) Cycloheximide (CHX) chase assay in parental MCF7 cells transduced with vector, HA-COPS5-WT or HA-COPS5-D151N. Cells were treated with CHX and then analysed by western blotting. (b) Half-life of NCoR protein was calculated by quantification of the western blots of anti-NCoR and anti-actin (for normalization). ${ }^{\star}$ Significant difference compared with other samples $(P<0.05$, Student's $t$-test). (c) Western blot analysis of COPS5, NCoR and ER $\alpha$ in MCF7 cells stably expressing empty vector (EV), WT or D151N COPS5 cDNA in the presence or absence of proteasome inhibitor MG132. (d) In vivo ubiquitination assay in HEK293T cells with overexpression of WT or D151N COPS5. Proteins pulled down by Ni-NTA beads and existed in whole cell lysates (WCL) are tested by western blot using antibodies shown on the left. His-Ubi, His ${ }_{6}$-tagged ubiquitin; Ubi-NCoR, polyubiquitinated NCoR protein. (e) Western blot analysis of NCoR expression on knockdown of COP9 subunits GPS1, CSN2 and CSN6 by specific shRNAs in 4OHT-resistant MCF7 clone \#3. Ctl, control. (f) Quantitative RT-PCR of mRNA of ER $\alpha$ target gene pS2 in the MCF7 cell lines shown in e in response to $40 H T(500 \mathrm{nM})$ for $48 \mathrm{~h}$. Data are presented as mean $+\mathrm{s} . \mathrm{e} . \mathrm{m}$. of three biological replicates. All of the $4 \mathrm{OHT}$-treated samples are significantly different from corresponding untreated samples $(P<0.01$, Student's $t$-test).

K48R ubiquitins reduced the overall ubiquitination levels, suggesting that both $\mathrm{K} 63$ and $\mathrm{K} 48$ are involved in polyubiquitination of NCoR (Supplementary Fig. 7). However, COPS5-WT only induced K63R-ubiquitination but lost its activity on K48R-ubiquitination, indicating that $\mathrm{K} 48$ is the major lysine residue which COPS5 regulates, though the E3 ligase(s) is yet to be identified (Supplementary Fig. 7). These data are in line with extensive evidence in the literature that proteasomal degradation-related poly-ubiquitination is mainly linked to ubiquitin-K48. To further evaluate the role of COP9 complex in COPS5-mediated NCoR protein degradation, we examined the role of some COP9 subunits by RNAi. In COPS5-overexpressing tamoxifen-resistant MCF7 clone \#3, RNAi-mediated knockdown of GPS1/CSN1 (two independent short hairpin RNAs (shRNAs)), CSN2 (one shRNA), COPS5 (two independent shRNAs) or CSN6 (two independent shRNAs) invariably caused induction of NCoR protein expression (Fig. 4e). Moreover, knockdown of these COP9 subunits led to restoration of tamoxifen-dependent transcriptional repression (Fig. 4f, Supplementary Fig. 8). Last, overexpression of WT, but not D151N, COPS5 decreased the levels of neddylated cullins in parental MCF7, whereas RNAi-mediated knockdown of COPS5 induced the neddylation of cullins in COPS5-overexpressed 4OHT-resistant MCF7 clone \#3 (Supplementary Fig. 9). These data indicate that COPS5 regulates neddylation of E3 ligase-associated cullins in breast cancer cells. Taken together, it is conceivable that mechanistically overexpression of COPS5 leads to degradation of NCoR protein through the ubiquitination-proteasomal pathway mediated by the COP9 complex in tamoxifen-resistant $\mathrm{ER} \alpha+$ breast cancer cells. 
Next we questioned if degradation of NCoR acts as the key step by which amplification or overexpression of COPS5 causes refractory to tamoxifen-treatment. To this end, we overexpressed NCoR complementary DNA (cDNA) in both parental and 4OHT-resistant MCF7 clone \#3. Apparently, transgenic overexpression of NCoR largely overcame the COPS5-mediated degradation and restored the NCoR protein level in clone \#3 (Fig. 5a). While E2 displayed a similar agonism in both parental and resistant clone \#3, 4OHT showed a robust agonistic activity on expression of five ER $\alpha$ target genes ( $p S 2$, GREB1, PGR, NRIP and IGFBP4) selectively in clone \#3 (Fig. 5b, Supplementary Fig. 10). Strikingly, re-expression of $\mathrm{NCoR}$ in clone \#3 restored the antagonistic 4OHT-mediated suppression of all tested ER $\alpha$ target genes (Fig. 5b, last two lanes, Supplementary Fig. 10). Knockdown of COPS5 led to induction of NCoR (but not ER $\alpha$ ) in clone \#3, which is antagonized by re-expression of exogenous COPS5 cDNA that was designed to avoid the recognition by shRNA, indicating that the shRNA is highly specific to COPS5 (Fig. 5c). Furthermore, knockdown of COPS5 restored the antagonistic/weak agonistic activity of $4 \mathrm{OHT}$ in clone \#3, whereas re-overexpression of COPS5 abolished the therapeutically beneficial activity again, using expression of five ER $\alpha$ target genes ( $p S 2$, GREB1, NRIP, IGFBP4 and CTSD) as biomarkers of ER $\alpha$-dependent transcription (Fig. 5d, Supplementary Fig. 11). Last, we knocked down NCoR in parental MCF7 cells by two independent shRNAs, which did not impact the expression of $\mathrm{ER} \alpha$ (Fig. 5e). Loss of NCoR strongly activated $\mathrm{ER} \alpha$ target gene expression ( $p S 2$, GREB1, NRIP, IGFBP4 and CTSD) on 4OHT-treatment, mimicking the resistance features observed in the spontaneous tamoxifen-resistant clones and engineered
COPS5-overexpressing cell lines (Fig. 5f, Supplementary Fig. 12). Notably, in TCGA $E R \alpha+$ breast cancer, deleterious mutations and deep deletion of NCOR1, the gene coding NCoR, were observed (Supplementary Fig. 13). And it has been shown that the NCoR/HDAC corepressor is required for 4OHT-mediated ER $\alpha$-dependent transcriptional repression ${ }^{25-27}$. These data strongly suggest that loss of NCoR protein expression by COPS5 amplification and overexpression is a driver of mediating tamoxifen-resistance.

To further understand the molecular mechanisms of COPS5/COP9 complex-mediated regulation of ER $\alpha$-dependent transcription, we conducted chromatin immunoprecipitation (ChIP) experiments on the pS2 gene promoter region surrounding the oestrogen-responsive elements (ERE). At basal conditions there was only minimal promoter occupancy of $E R \alpha$, corepressor NCoR, coactivator PCAF, and COP9 subunits COPS5 and CSN2 in both parental and 4OHT-resistant MCF7 cells (Fig. 6a,b, white bars). In the presence of E2, ER $\alpha$ and PCAF bound to the $p S 2$ promoter as shown previously ${ }^{28,29}$. By contrast, COP9 subunits (COPS5/CSN2) and NCoR were not recruited (Fig. 6a,b, grey bars), indicating that COP9 and NCoR are not directly involved in transcriptional activation by promoter modulation. Surprisingly, on treatment with antagonist 4OHT in parental MCF7 cells, $\mathrm{ER} \alpha, \mathrm{NCoR}$, COPS5 and CSN2, but not PCAF, coincidentally occupied the $p S 2$ promoter, which suggests that basal level of COP9 may directly regulate NCoR on the promoter. However, in 4OHT-resistant MCF7 cells, neither NCoR nor COP9 was recruited, whereas ER $\alpha$ and PCAF were able to bind to the promoter on 4OHT-treatment (Fig. 6a,b, black bars), due probably to global loss of NCoR by overexpression of

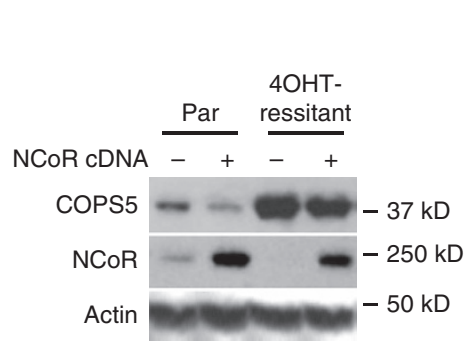

b

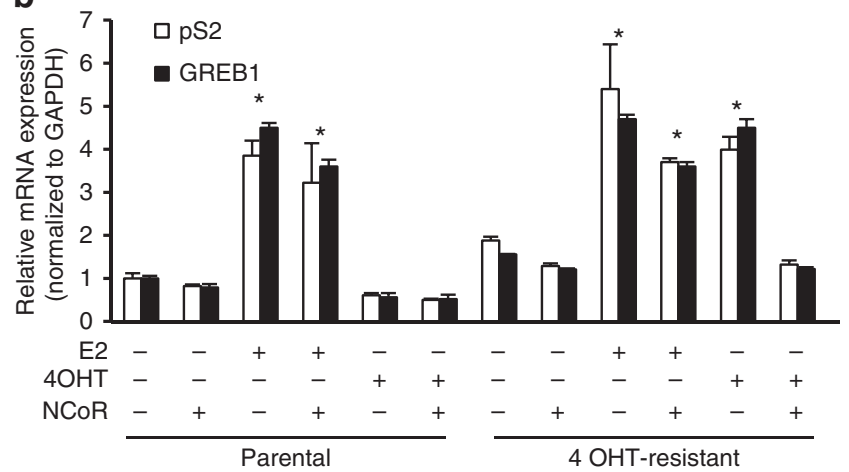

C

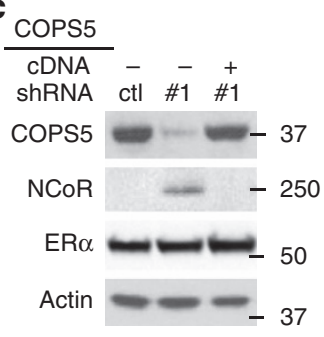

d

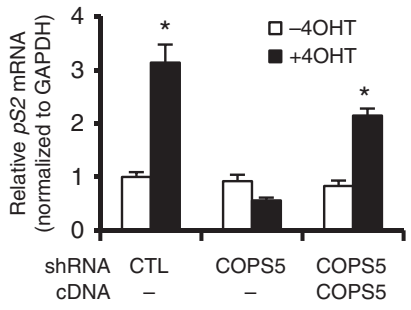

f

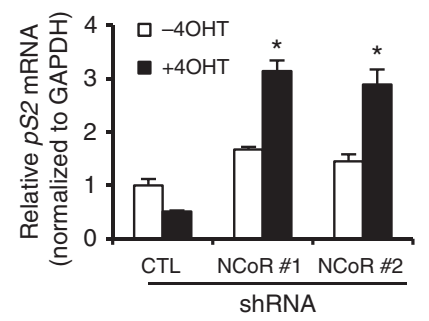

Figure 5 | COPS5-mediated 40HT-resistance is dependent on NCoR downregulation. (a) Western blot analysis of COPS5 and NCoR in MCF7 cells with transient expression of COPS5 and/or NCOR. (b) Quantitative RT-PCR analysis of ligand-regulated ER $\alpha$ target gene expression in parental and 4OHT-resistant MCF7 clones with expression of NCoR. ${ }^{*}$ Significant difference compared with corresponding untreated basal levels ( $P<0.01$, Student's $t$-test). (c) Western blot analysis of COPS5, NCoR and ER $\alpha$ expression in the 4OHT-resistant MCF7 cells stably transduced with COPS5 shRNA or rescuing COPS5 cDNA. (d) Quantitative RT-PCR analysis of ER $\alpha$ target gene $p S 2$ in the MCF7 cell lines shown in c in response to $40 H T$ (500 nM, $48 \mathrm{~h}$ ). *Significant difference compared with the untreated CTL sample ( $P<0.01$, Student's t-test). (e) Western blot analysis of NCoR and ER $\alpha$ expression on knockdown by stable NCoR shRNAs in parental MCF7 cells. (f) Quantitative RT-PCR analysis of ER $\alpha$ target gene $p S 2$ in the MCF7 cell lines shown in e in response to $4 \mathrm{OHT}(500 \mathrm{nM}, 48 \mathrm{~h})$. ${ }^{\star}$ Significant difference compared with the untreated CTL sample $(P<0.01$, Student's $t$-test). Data are presented as mean + s.e.m. of three biological replicates. CTL, control. 

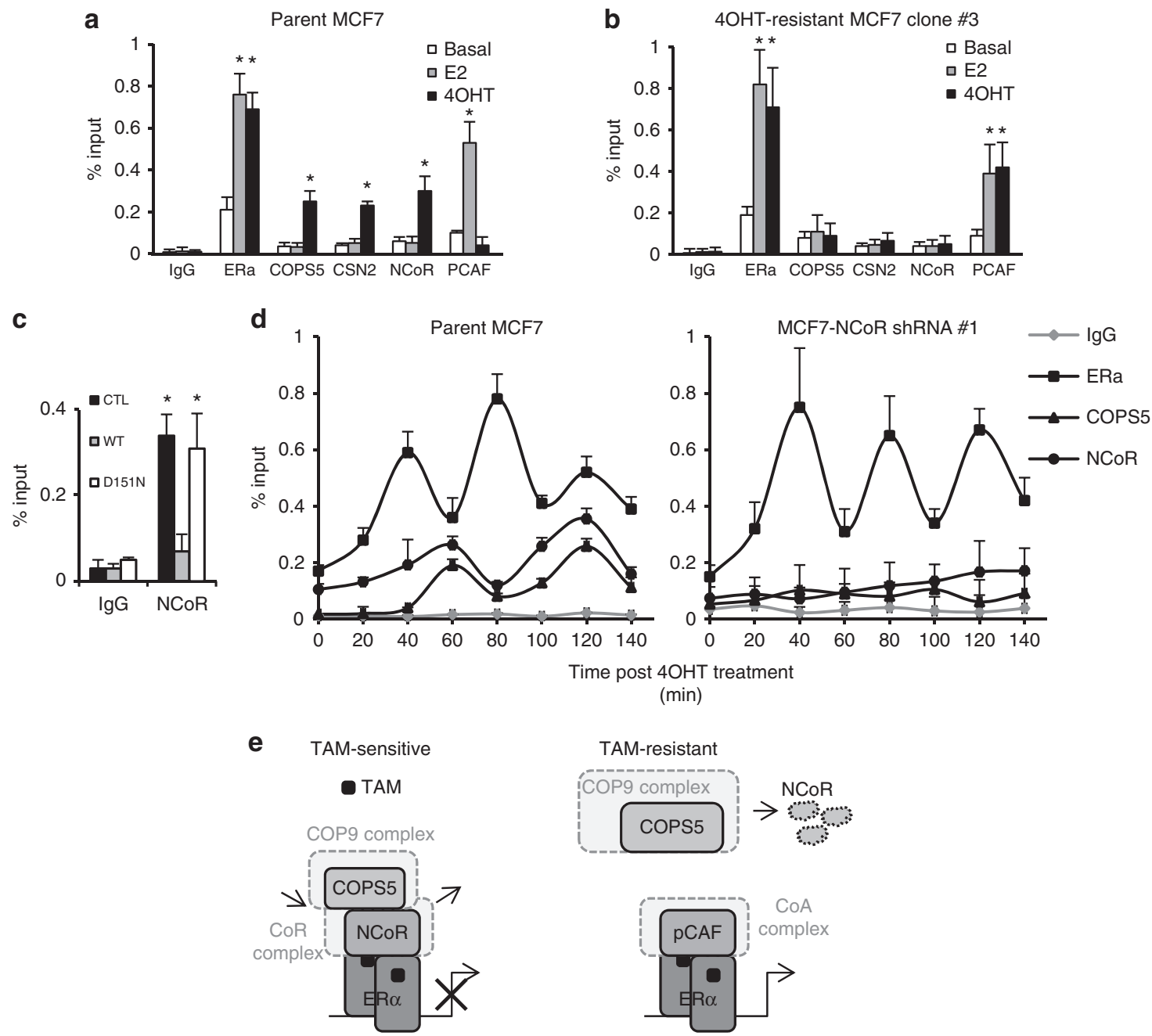

Figure 6 | COPS5 overexpression alters the dynamics of coregulator exchange on the $\mathbf{p S 2}$ promoter. ChIP analysis of the pS2 promoter occupancy of $\mathrm{ER} \alpha, \mathrm{COPS} 5, \mathrm{CSN} 2, \mathrm{NCOR}$ and PCAF on stimulation with ER $\alpha$ ligands for $2 \mathrm{~h}$ in (a) parental MCF7 cells and (b) $4 \mathrm{OHT}$-resistant MCF7 clone \#3. E2, $1 \mathrm{nM}$; $4 \mathrm{OHT}, 1 \mu \mathrm{M}$. Data are presented as mean + s.e.m. from three independent experiments. ${ }^{\star}$ Significant difference compared with corresponding basal recruitment ( $P<0.01$, Student's t-test). (c) ChIP analysis of NCoR on stimulation with ER $\alpha$ ligands for $2 \mathrm{~h}$ in parental and engineered COPS5-overexpression MCF7 cells. *Significant difference compared with corresponding basal recruitment $(P<0.01$, Student's $t$-test). (d) Kinetic ChIP analysis of ER $\alpha$, COPS5 and NCoR on the pS2 promoter at 20-min interval in parental MCF7 or NCoR shRNA \#1 cells. Data are presented as mean + s.d. from two independent experiments. (e) A schematic representation of the proposed molecular model of functional interactions between COPS5 and NCoR in ER $\alpha$-dependent transcription based on the ChIP data (see text for details). TAM, tamoxifen.

COPS5. We also confirmed a loss of NCoR recruitment on the pS2 promoter on engineered overexpression (Fig. 4c) of WT but not D151N COPS5 in MCF7 cells (Fig. 6c). We further performed kinetic ChIP experiments, which revealed that there was a cyclical sequential promoter recruitment of $\mathrm{ER} \alpha, \mathrm{NCoR}$ and COPS5 after 4OHT-treatment in parental MCF7 cells (Fig. 6d, left panel). Interestingly, the on/off cycles of ER $\alpha$ and NCoR/COPS5 are operated at different rate, which would suggest that COPS5 occupancy is associated with $\mathrm{NCoR}$ rather than ER $\alpha$. Indeed, when we knocked down NCoR by RNAi in parental MCF7 cells, the $\mathrm{ER} \alpha$ cyclical recruitment was not impacted whereas the recruitment of COPS5 was completely abolished (Fig. 6d, right panel). This also indicates that promoter occupancy of basal COP9/COPS5 is NCoR-dependent, supporting the protein interaction and gene expression data shown above. In summary, based on the ChIP data, we proposed a model mechanism by which NCoR and COPS5 functionally interact in orchestrating the $\mathrm{ER} \alpha$-dependent transcription: (1) in tamoxifen-sensitive breast cancer cells, tamoxifen triggers the recruitment of $\mathrm{ER} \alpha / \mathrm{NCoR}$ corepressor transcriptional apparatus.
Low basal level of COP9/COPS5 is associated with NCoR and constantly degrade/recycle the NCoR complex as part of the repression strategy to prevent coactivator recruitment, similar to the mechanism of cyclical activation ${ }^{28,29}$. Therefore, tamoxifen retains as an antagonist; (2) in tamoxifen-resistant cells, NCoR is globally diminished by degradation due to COPS5 overexpression and consequently high levels of COP9/COPS5 activity. In the absence of NCoR, COP9/COPS5 is no longer recruited by tamoxifen-treatment. Instead, the coactivator complex (PCAF as tested) occupies the ER $\alpha$-dependent transcriptional promoters/enhancers, leading to drug resistance via a conversion of $4 \mathrm{OHT}$ to an agonist (Fig. 6e).

Inhibition of COPS5 restores tamoxifen sensitivity. Finally, we investigated the cancer biological relevance and therapeutic potentials of COPS5-related molecular mechanisms in endocrine-refractory breast cancer. Using the doxycyclineinducible shRNA system, we established stable cell lines with shRNAs targeting COPS5 and COPS5 cDNAs in 4OHT-resistant 
a

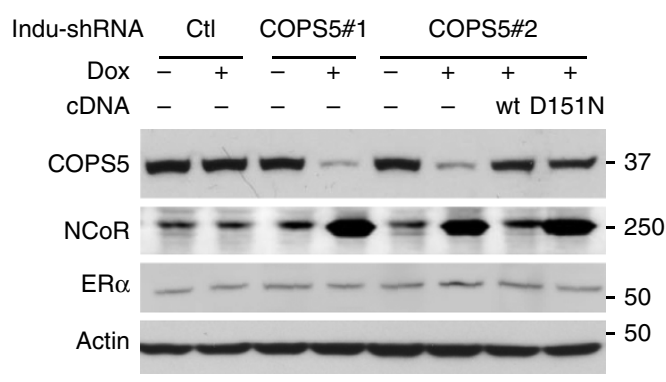

\section{b}

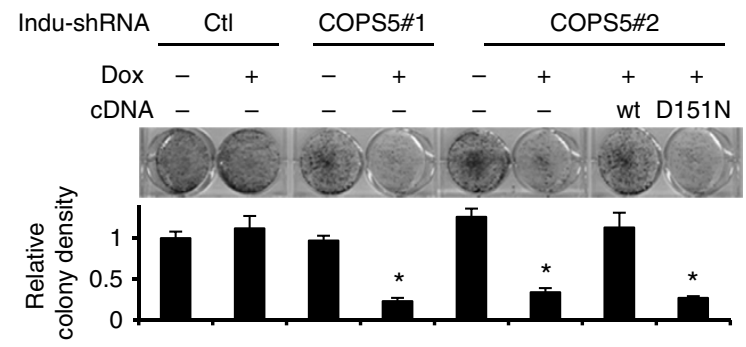

C
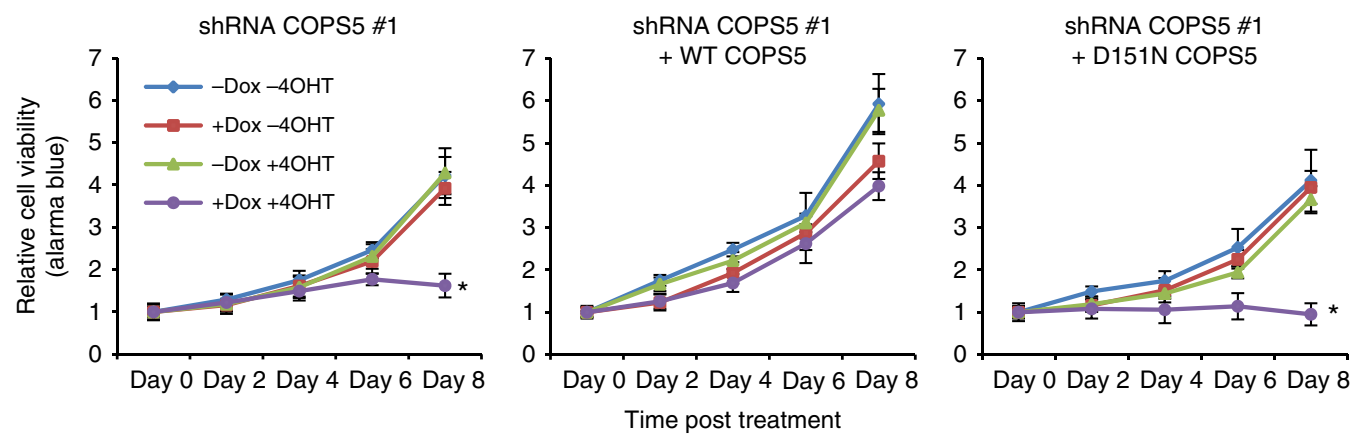

d
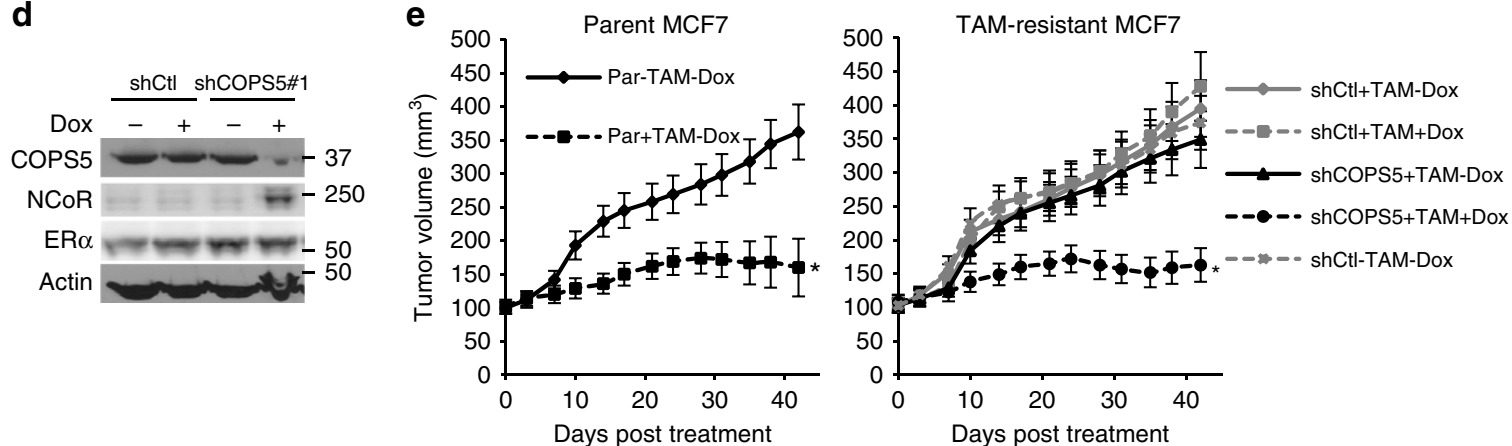

Figure 7 | Genetic inhibition of COPS5 re-sensitizes tamoxifen-resistant breast cancer cells to endocrine therapy. (a) Western blot analysis of doxycycline (Dox)-inducible shRNA-mediated knockdown of COPS5 and complementary re-expression of COPS5 cDNAs in the cultured $40 H T$-resistant MCF7 clone \#1. (b) Colony formation assay of 4OHT-resistant MCF7 clone \#1 on knockdown by COPS5 shRNAs and rescue by COPS5 cDNAs after $4 \mathrm{OHT}$-treatment for 14 days at $1 \mu \mathrm{M}$. *Significant difference compared with basal growth $(P<0.01$, Student's $t$-test). (c) Growth of $4 \mathrm{OHT}$-resistant MCF7 clone \#3 in response to COPS5 knockdown and 4OHT-treatment $(1 \mu \mathrm{M})$. Data are presented as mean + s.e.m. from three independent experiments. *Significant difference compared with other samples $(P<0.01$, Student's $t$-test). (d) Western blot analysis of the xenograft tumours derived from 4OHT-resistant MCF7 clone \#3. Cells were stably engineered with doxycycline-inducible shRNAs. (e) Volumes of the MCF7 xenograft tumours on treatments as indicated. Data are presented as mean \pm s.d. of five mice each group. ${ }^{*}$ Significant difference compared with other samples $(P<0.01$, Student's $t$-test). Ctl, control; DOX, doxycycline; Indu-shRNA, inducible shRNA; Par, parental; TAM, tamoxifen.

MCF7 cells (Fig. 7a). When COPS5 was knocked down by RNAi, an upregulation of NCoR expression can be clearly observed as we showed above (Figs $4 \mathrm{e}, 5 \mathrm{c}$ and $7 \mathrm{a}$ ). Crucially, COPS5-WT but not D151N was able to abolish the shRNA-mediated upregulation of NCoR (Fig. 7a), confirming the importance of catalytic activity of COPS5 in regulating NCoR protein expression. ER $\alpha$ protein expression was not affected by any of these genetic modulations (Fig. 7a). Moreover, these cells were proliferating similarly with or without 4OHT-treatment when COPS5 was maintained at high level using control shRNA. By contrast, knockdown of COPS5 by two independent shRNAs re-sensitized the cells to tamoxifen-treatment. Importantly, expression of a shRNAresistant WT COPS5 cDNA was able to completely rescue the growth inhibition induced by shRNA, while the isopeptidase-deficient D151N failed to do so (Fig. 7b), indicating that the catalytic activity of COPS5 is essential to confer tamoxifen-resistance in breast cancer cells. In time-course studies,
COPS5 shRNA exhibited growth inhibition of the resistant cells only in the presence of 4OHT. Again, this phenotype was rescued by WT COPS5 but not the D151N mutant (Fig. 7c). It is therefore conceivable that combination of tamoxifen and a COPS5 inhibitor would be a desired therapeutic approach to overcoming tamoxifen-resistance in breast cancer. Last, we tested the in vivo role of COPS5 in mediating tamoxifen-refractory. Using the MCF7 xenograft model, we first confirmed that induction of COPS5 shRNA, but not the control shRNA, led to substantial knockdown of COPS5 expression after doxycycline-treatment in vivo (Fig. 7d). Subsequently, only NCoR expression was upregulated whereas ER $\alpha$ did not change (Fig. 7d). As expected, the tumours derived from parental MCF7 cells were sensitive to tamoxifen-treatment, as shown extensively in the literature. Dosing tamoxifen at $100 \mathrm{mg} \mathrm{kg}^{-1}$ three times per week induced a substantial stasis of tumour growth (Fig. 7e). The tumours derived from the 4OHT-resisatant MCF7 clone \#3 were 
completely resistant to the same regimen though the basal growth (vehicle-only) was similar to the parental tumours. However, knockdown of COPS5 by inducible shRNA restored the sensitivity to tamoxifen-treatment in the COPS5-overexpressed resistant tumours (Fig. 7e). Satisfyingly, COPS5 reduction and subsequent NCoR upregulation by COPS5 shRNA were observed at the end point of doxycycline/tamoxifen-treatment at day 42 in vivo (Supplementary Fig. 14, last two lanes). Furthermore, ER $\alpha$ expression was not changed in these MCF7 tumour samples (Supplementary Fig. 14). Interestingly, while knockdown of an essential gene PLK1 induced a remarkable growth inhibition of the subcutaneous tumours of parental MCF7 cells, neither knockdown nor overexpression of COPS5 showed a significant impact on MCF7 tumour growth (Supplementary Fig. 15). It suggests that the dependence on COPS5 is selective in COPS5-amplified/overexpressed MCF7 cells on tamoxifentreatment. These results support that COPS5 is a therapeutic target in COPS5-amplified, tamoxifen-resistant breast cancer.

\section{Discussion}

Drug resistance represents a major clinical challenge in targeted therapy for cancer ${ }^{30,31}$. In $\mathrm{ER} \alpha$-positive breast cancer, although most of the patients initially responded well to tamoxifen or other endocrine treatment, eventually many of them inevitably developed to drug-resistant and metastatic disease which is incurable ${ }^{8}$. Although a number of resistance mechanisms have been proposed, most of them failed to translate into clinical benefits to overcoming the resistance. In this report, we revealed a novel genomics-driven mechanism that renders resistance to tamoxifen, namely amplification and overexpression of the gene encoding COPS5, a component of the neddylation-catalyzing COP9 signalosome complex regulating the activity of SCF E3 ligases. In the TCGA primary tumour cohort, amplification of COPS5 is relatively focal and correlates with high COPS5 mRNA expression (Fig. 1). It is also evident that overexpression of COPS5 is further enriched in tamoxifen-resistant tumours (Fig. 1). Since (1) the tamoxifen-refractory tumour samples used for IHC were biopsied/autopsied from patients who had been treated with tamoxifen for at least 5 months to $>10$ years; and (2) short-term treatment of MCF7 cells with tamoxifen in vitro or in vivo did not alter the COPS5 protein expression (Supplementary Fig. 16), the elevated COPS5 expression is probably due to drug resistance-associated selection (genomic amplification) rather than acute induction by tamoxifen. Interestingly, overexpression of COPS5 induces ubiquitination and proteasomal degradation of the NCoR protein, a key corepressor of tamoxifen-bound $\mathrm{ER} \alpha$, leading to de-repression of ER $\alpha$ target genes in resistant breast cancer (Figs 2-5). Based on the data we showed in this report, in hormone-sensitive $\mathrm{ER} \alpha$-positive breast cancer normal basal levels of COPS5/COP9 precisely control the protein stability of ER $\alpha$ corepressor NCoR. In the presence of antiestrogen drugs, COP9/COPS5 is associated with $\mathrm{NCoR}$ on the target gene promoter and constantly degrades and recycles the NCoR complex as a molecular strategy of transcriptional repression, which is similar to the previously reported mechanism of $\mathrm{ER} \alpha$ activation through the cyclical clearance of coregulators ${ }^{28,29}$. Therefore, the drugs are able to suppress $E R \alpha$-regulated transcription and consequently inhibit cancer cell growth. However, when COPS5 is amplified and overexpressed in endocrine-resistant cancer cells, elevated COPS5/COP9 activity induces global NCoR protein degradation. As a result, tamoxifen recruits the coactivator complex (PCAF as tested) instead to the ER $\alpha$-dependent transcriptional promoters/ enhancers, leading to drug resistance via a conversion of tamoxifen to an agonist (Fig. 6e).
The functional relevance of NCoR is further supported by the fact that NCoR is deleteriously mutated in about $5 \%$ of ER $\alpha$-positive breast cancer (TCGA, Supplementary Fig. 13) ${ }^{32,33}$. Furthermore, downregulation of NCoR mRNA expression in breast cancer patients correlates with shorter relapse ${ }^{34}$, suggesting its crucial role in breast cancer development. More importantly, we showed that loss of NCoR expression is sufficient to trigger the resistance to tamoxifen, in line with the previous reports. We revealed a switch of transcription coregulators on the promoters of $\mathrm{ER} \alpha$ target genes on overexpression of COPS5 and loss of NCoR (Fig. 6e). NCoR is replaced by coactivators including PCAF by tamoxifen-treatment in endocrine-resistant breast cancer cells, leading to activation of the $\mathrm{ER} \alpha$ signalling which is required for the cancer cell growth. In fact, a number of endocrine-resistance mechanisms may involve the $\mathrm{ER} \alpha$-dependent transcription, for example, hotspot mutations in $\mathrm{ER} \alpha$ ligand-binding domain, NCOA1 amplification, deleterious mutations in NCoR and PI3K activation. This $\mathrm{ER} \alpha$-centric resistance mechanism implicates a possible crosstalk between COPS5 amplification with other aberrant alterations associated with endocrine-resistance. Here we uncovered the COPS5-NCoR-coactivator axis as a key mediator in conferring the drug resistance. Of note, breast cancer cells with overexpression of COPS5 appeared to be less sensitive to the SERD fulvestrant in vitro as well (Supplementary Fig. 2), suggesting that it could be a common resistance mechanism to endocrine therapies. Because ER $\alpha$ should be degraded by fulvestrant, the exact molecular mechanism by which COPS5 can induce fulvestrant-resistance is still ambiguous and currently under investigation.

The COP9 protein complex consists of eight subunits in which COPS5 is the only catalytically active metalloprotease. The complex is well-characterized as a master regulator in ubiquitin-mediated protein degradation ${ }^{35}$. COP9 exerts these activities through regulating cullin-RING ligases (CRLs) and RING-containing ubiquitin ligases that target many proteins for ubiquitin/proteasome-mediated degradation. COPS5-mediated removal of Nedd8 (deneddylation) from cullin is a crucial step for CRLs' activity. COPS5 possesses a JAMM (JAB1/MPN/ MOV34) metalloprotease motif $\left(\mathrm{EX}_{n} \mathrm{HXHX}_{10} \mathrm{D}\right)$ that is required for cullin deneddylation. It has been shown that COP9/COPS5 can regulate the stability of a variety of cancer-related proteins including p27, p53, MDM2, c-Jun and key proteins involved in DNA damage repair. Consequently its role in tumorigenesis has been proposed even though there is a lack of clear mechanistic understanding ${ }^{36-38}$. Accumulating evidence suggests critical roles of COPS5 in different stages of breast cancer development. A number of studies have demonstrated that COPS5 is overexpressed in breast cancer ${ }^{39-41}$. The involvement of COPS5 in breast cancer, particularly ER $\alpha$-negative tumours, appears to be linked to the EGFR and HER2 pathways by mediating downstream signalling that contributes to the progression of tumour growth ${ }^{40,41}$. In addition, COPS5 has been shown to be involved in breast cancer initiation by coordinating the epithelial transformation evoked by Myc and Ras in mouse models ${ }^{42}$. It was also proposed that ligand-induced $\mathrm{ER} \alpha$ degradation was regulated by COPS5 in hormone-dependent breast cancer cells ${ }^{24}$. Here using integrated genomic and functional approaches, we discovered a key role of COPS5/COP9 in conferring resistance to endocrine therapy. We revealed that $\mathrm{NCoR}$ is a substrate of COPS5/COP9 that is crucial in mediating endocrine-resistance in breast cancer. It is conceivable that COPS5 might be involved in multiple stages of breast cancer development by regulating different protein targets via its isopeptidase activity. Further identification and elucidation of the E3 ligase that connects COPS5/COP9 and NCoR are of importance to understand the 
precise mechanisms underlying endocrine resistance and identify additional druggable molecular targets.

Our findings also offer therapeutic opportunities to overcome tamoxifen-resistance. Since the $\mathrm{Zn}^{2+}$-dependent metalloprotease activity appears to be critical in the resistance mechanism (Fig. 7), inhibition of the catalytic activity of COPS5 is an intriguing and promising strategy for drug discovery. Alternatively, based on recent finding on the structure of eight-unit COP9 complex $^{43}$, it is possible to modulate COPS5 activity by tackling the interactions between COP9 subunits, particularly the interaction between COPS5 and COPS6/CSN6 subunits that is crucial for maintaining the isopeptidase activity. The major function of the isopeptidase/metalloprotease activity of COPS5/COP9 is deneddylation of cullin proteins in SCF/CRL ubiquitin ligases to trigger the neddylation cycles for maintaining the ligase activity. Consequently, using inhibitors of Nedd8-activating enzymes, for example, MLN4924, might have an impact on the COPS5/NCoR axis ${ }^{44}$. Last, there are kinases like CK2 and PKD that are associated with the COP9 complex and crucial for its activity ${ }^{45}$. Modulation of the kinase activity, which is generally more feasible for drug discovery, could be an approach to inhibiting the COPS5 activity as well. Regardless how the COPS5 inhibitors will be developed, our data suggest that combination with $\mathrm{ER} \alpha$ antagonists is probably needed to re-sensitize the refractory tumours to endocrine therapy. In summary, the current work supports the idea of developing COPS5 inhibitors to treat tamoxifen-resistant $\mathrm{ER} \alpha+$ breast cancer patients harbouring COPS5 amplification and/or overexpression.

\section{Methods \\ Cell lines and reagents. MCF7 and T47D were purchased from ATCC. HEK293FT was purchased from Life Technologies. Tamoxifen-resistant MCF7 clones were generated by dose-escalation 4OHT-treatment in phenol red-free RPMI1640 culture medium supplied with 10\% charcoal-stripped FBS (Life Technologies): $200 \mathrm{nM}$ for 2 weeks, $500 \mathrm{nM}$ for 2 weeks and then $2 \mu \mathrm{M}$ for 4 weeks. Single clones were picked up under microscope and then expanded in $2 \mu \mathrm{M} 4 \mathrm{OHT}$ - containing phenol red-free RPMI1640 culture medium supplied with $10 \%$ charcoal-stripped FBS. Antibodies used in this report were: COPS5 (A300-014A, Bethyl Laboratories), NCoR (PA1-844A, Thermo Scientific) for western blot (WB) and IHC, NCoR (sc-1609, Santa Cruz) for IP and ChIP, ER $\alpha$ (SC-543, Santa Cruz) for WB, ER $\alpha$ (61035, Active Motif) for ChIP, GPS1 (ab4535, Abcam), CSN2 (PA1-24686, Thermo Scientific), CSN6 (sc-47965, Santa Cruz), NEDD8 (Y297) (ab81264, Abcam), PCAF (sc-13124, Santa Cruz), HDAC1 (sc-6298, Santa Cruz), HDAC3 (sc-17795, Santa Cruz), HA-tag (MMS-101P, Covance), His-tag (sc-803, Santa Cruz), actin (A-3853, Sigma), tubulin (T6074, Sigma) and secondary antibodies were purchased from Sigma. Compounds used were tamoxifen (T5648, Sigma), 4OHT (94873, Sigma), E2 (E8875, Sigma), fulvestrant (I4409, Sigma), flavopiridol (S2679, Selleckchem) and MG132 (S2619, Selleckchem).}

Lentiviral RNAi and cDNA. The inducible shRNA vector was modified from the previous reports ${ }^{46,47}$. Target sequences are listed below: COPS5 \#1: $5^{\prime}$-GTCTCAG GTTATTAAGGATAA-3 ${ }^{\prime}$, COPS5 \#2: 5' -CAGTCTCTGAGAAGTACTTTA-3', NCoR \# 1: 5' -GCTCTCAAAGTTCAGACTCTT-3', NCoR \#2: 5'-GCCCACAGA TGATGAAGAAAT-3', GPS1 \#1: 5'-CCGAGACATCATCTTCAAATT-3', GPS1 \# 2: 5' -CGCTGCCGGTTCAGGTGTTTA-3', CSN2 \#1: 5'-GCACTCTATGAA CAGTCACTT-3', CSN6 \#1: 5'-CGTGGAAGAGAAGATTATCAT-3', CSN6 \#2: $5^{\prime}$-CAGTTTGTGAACAAGTTCAAT- $3^{\prime}$. Lentiviruses were packaged by cotransfecting $1 \times 10^{7}$ HEK293FT cells with $5 \mu \mathrm{g}$ of shRNA-encoding plasmid, $1.5 \mu \mathrm{g}$ each of $\Delta 8.91$ packaging plasmid, pCMV-VSV-G envelop plasmids using TransIT293 (Mirus). Cell culture media was changed after $24 \mathrm{~h}$ and lentivirus-containing supernatant was harvested $72 \mathrm{~h}$ post transfection. Infection of the target cells was carried out with a final concentration of $8 \mu \mathrm{g} \mathrm{ml}{ }^{-1}$ polybrene-containing viral medium and selected in $1 \mu \mathrm{g} \mathrm{ml}^{-1}$ puromycin (for shRNA or cDNA vector) or $200 \mu \mathrm{g} \mathrm{ml}^{-1}$ neomycin (for cDNA vector) after $48 \mathrm{~h}$ post infection.

WB analysis. Five per cent non-fat milk in TBS-T $(0.1 \%$ tween 20$)$ was used as blocking and probing buffer. TBS-T was used as wash buffer. Cells were harvested by scraping into ice-cold PBS and lysed using RIPA buffer $(150 \mathrm{mM} \mathrm{NaCl}, 0.5 \%$ Triton X-100, $1 \%$ sodium deoxycholate, $0.1 \%$ SDS, $1 \mathrm{mM}$ EDTA and $50 \mathrm{mM}$ Tris- $\mathrm{HCl}, \mathrm{pH}$ 7.4) supplemented with protease inhibitor cocktails (Roche). Antibodies were probed using manufacturer's recommended conditions. Signals were detected using the ECL detection system (GE Healthcare). NIH Image) software was used for quantification of WBs. Uncropped scans were shown in Supplementary Fig. 17

Quantitative RT-PCR. Total RNA was purified using the RNeasy kit (Qiagen), and treated with DNase before cDNA preparation. cDNA was synthesized following manufacturer's manual (SuperScript III RT, Life Technologies). mRNA levels were determined using TaqMan Probe-Based Gene Expression Assays using ABI PRISM Detection System (Applied Biosystems/Life Technologies).

All primers and probes of genes tested in this report were purchased from Life Technologies. VIC-MGB GAPDH control primers and probe were examined as internal controls. All samples were tested in triplicate and normalized to GAPDH levels. Gene-specific relative mRNA levels were calculated by the standard equation $2^{\text {( } \Delta \mathrm{CT} \text { sample }-\Delta \mathrm{CT} \text { control)} \text {. }}$

Copy number. TaqMan qPCR copy number assay (Life Technologies) was used to measure the copy numbers of COPS5 in genomic DNA following the protocol from the vendor. LINE1 was used as the internal normalization control.

Immunoprecipitation. NETN buffer (25 mM Tris-HCl, pH 8.0, $150 \mathrm{mM} \mathrm{NaCl}$ $1 \mathrm{mM}$ EDTA, $1 \%(\mathrm{v} / \mathrm{v})$ Nonidet P-40, $10 \%(\mathrm{v} / \mathrm{v})$ glycerol, $1 \times$ protease inhibitor mix) was used for lysis and immunoprecipitation. Immunoprecipitation was performed using standard protocol ${ }^{48}$.

Chromatin immunoprecipitation. Chromatin extraction (ab117152) and ChIP kits (ab117138) were purchased from Abcam. ChIP was conducted following the instructions of vendor. Primers used for pS2-ERE promoter are: forward: $5^{\prime}$-GCT TAGGCCTAGACGGAATGGGC-3' ; reverse: $5^{\prime}$-CCAGGTCCTACTCATATCT GAGAG- $3^{\prime}$.

CHX chase experiment. MCF7 cells are infected with COPS5-WT- or D151N-expressing lentivirus, and $16 \mathrm{~h}$ later selected by puromycin $\left(2 \mu \mathrm{g} \mathrm{ml}^{-1}\right)$ for $24 \mathrm{~h}$. The cells were then treated with $20 \mu \mathrm{g} \mathrm{ml}^{-1}$ of CHX for $0.5-4 \mathrm{~h}$, and cell lysates were isolated for WB analysis.

In vivo ubiquitination assay. HEK293T cells were co-transfected with plasmids encoding His6-tagged ubiquitin and NCoR, and harvested $36 \mathrm{~h}$ after transfection. The cells were lysed in $6 \mathrm{ml}$ of guanidinine lysis buffer (6 M guanidinium$\mathrm{HCl}, 100 \mathrm{mM}$ Na2HPO4/NaH2PO4, $10 \mathrm{mM}$ Tris, $\mathrm{pH} \mathrm{8,} 5 \mathrm{mM}$ imidazole and $10 \mathrm{mM} \beta$-mercaptoethanol). Then $100 \mu \mathrm{l}$ of $\mathrm{Ni} 2+$-nitrilotriacetic acid-agarose beads (Qiagen) was added into cell lysates, followed by incubation overnight. The beads were then washed with the following buffers: guanidinine buffer $(6 \mathrm{M}$ guanidinine- $\mathrm{HCl}, 100 \mathrm{mM}$ Na2HPO4/NaH2PO4, $10 \mathrm{mM}$ Tris- $\mathrm{HCl}, \mathrm{pH} 8,10 \mathrm{mM}$ $\beta$-mercaptoethanol), urea buffer (8 M urea, $100 \mathrm{mM} \mathrm{Na} 2 \mathrm{HPO} 4 / \mathrm{NaH} 2 \mathrm{PO} 4,10 \mathrm{mM}$ Tris- $\mathrm{HCl}, \mathrm{pH} 8,10 \mathrm{mM} \beta$-mercaptoethanol), buffer A ( $8 \mathrm{M}$ urea, $100 \mathrm{mM}$ $\mathrm{Na} 2 \mathrm{HPO} 4 / \mathrm{NaH} 2 \mathrm{PO} 4,10 \mathrm{mM}$ Tris- $\mathrm{HCl}, \mathrm{pH} 6.3,10 \mathrm{mM} \beta$-mercaptoethanol) and buffer A plus $0.1 \%$ Triton X-100. Elution was carried out with $200 \mathrm{mM}$ imidazole in $150 \mathrm{mM}$ Tris- $\mathrm{HCl}$ (pH 6.7) supplemented with 5\% SDS, 30\% glycerol and $500 \mathrm{mM} \beta$-mercaptoethanol. The eluates were diluted 1:1 with $2 \times$ SDS-PAGE sample buffer and subjected to SDS-PAGE, and ubiquitinated proteins probed with specific antibodies by WB analysis.

Cell viability assays. In vitro cell growth and viability assays were performed by the alamarBlue Cell Viability Assay kit (Life technologies) following the vendor's instruction. Colony formation experiments were conducted using crystal violet staining ${ }^{49}$. Quantification of colony density was measured by dissolving crystal violet using $10 \%$ acetic acid for $20 \mathrm{~min}$, and then followed by measurement of optical density at $590 \mathrm{~nm}$.

Acquisition and analysis of TCGA data. Data were downloaded from the TCGA data portal (https://tcga-data.nci.nih.gov/tcga/tcgaDownload.jsp) and visualized or analysed by Excel (Microsoft) and/or the cBio visualization portal (http://www.cbioportal.org/).

Clinical samples. From January 2005 to December 2014, the tissues of breast cancer patients with tamoxifen-refectory ( $n=30$; age range, $21-70$ years) and the tissues of tamoxifen-naive breast cancer as the controls $(n=31$; age range, 20-73 years) were collected, respectively. The cohorts were closely matched for age enroled into this study $(P>0.05)$. The patients' medical records were retrospectively reviewed to collect diagnostic information. All subjects, enroled in this study at the Fudan University Shanghai Cancer Center (Shanghai, China), gave written informed consent. The Institute Ethical Committee approved the study protocol according to the guidelines of Helsinki conventions.

Immunohistochemistry. IHC staining for COPS5 and NCoR was performed on formalin-fixed paraffin-embedded tissues using a protocol as previously 
described ${ }^{50}$. COPS5 was detected with a commercially available rabbit polyclonal antibody at a dilution of 1:200. To evaluate NCoR expression, we used rabbit polyclonal antibody PA1-844A (dilution 1:400). Anti-ER $\alpha$ antibody (dilution 1:500, GeneTex Inc) was used for IHC of ER $\alpha$. IHC staining was assessed by two independent pathologists under blinded conditions. The concordance between scores from different cores of the same tumour was $>90 \%$. The intensity of COPS5 and NCoR staining was evaluated using the following criteria: we regarded close to $100 \%$ of cells exhibiting strong nuclear staining as a very strong positive result $(+++)$; $>75 \%$ of cells exhibiting strong cytoplasmic and/or nuclear staining as a strong positive result $(++)$; and $50-75 \%$ of cells exhibiting strong cytoplasmic and/or $10-75 \%$ of cells with nuclear staining as a positive result $(+)$, whereas weak cytoplasmic expression (to any extent) in $<50 \%$ or $<10 \%$ nuclear staining of cells was considered as negative expression $(-)^{51}$.

Animal experiments. All animal studies were performed under regulations provided by the Zhejiang Provincial Experimental Animal Regulation Committee and the Shanghai Municipal Experimental Animal Regulation Committee. Ten million MCF7 cells were inoculated s.c. in 4-6-week-old female, intact athymic nude mice. Estradiol pellets $(0.5 \mathrm{mg}$ per pellet) were implanted s.c. into the right back skin between the ear and shoulder. When the tumours were palpable $\left(\sim 100 \mathrm{~mm}^{3}\right)$, mice were randomized into treatment groups (five animals per group). For inducing shRNA expression, animals received doxycycline hyclate $\left(40 \mathrm{mg} \mathrm{kg}^{-1}\right.$ per day by oral gavage) for the duration of the study. Tamoxifen-treatment ( $20 \mathrm{mg} \mathrm{kg}^{-1}$ of tamoxifen citrate in peanut oil) was administered s.c. daily Monday through Friday for the duration of the study. Vehicles were dosed in control groups. Tumour volume and body weight were measured twice per week. Tumour volume was determined by using calipers for measurement of the longest (Length) and shortest (Width) dimensions and calculated as (Length $\times$ Width $\left.^{2}\right) / 2$. To monitor shRNA knockdown efficiency, a separate group of tumour tissues were collected after 2 weeks of doxycycline-treatment and snap-frozen in liquid nitrogen for WB analysis.

Statistics. Tumour size comparisons between groups were done using one-way analysis of variance. In vitro cell growth data, transcription quantitative reverse transcription-PCR (qRT-PCR) data and other biological assay data were compared between two groups using Student's $t$-test (two-tailed). Tumour sample expression data and IHC staining data were analysed by Pearson's $\chi^{2}$-test. For all tests, the level of significance was set at $P<0.05$.

Data availability. The authors declare that all other data supporting the findings of this study are available within the article and its Supplementary Information files.

\section{References}

1. Burstein, H. J. et al. Adjuvant endocrine therapy for women with hormone receptor-positive breast cancer: american society of clinical oncology clinical practice guideline focused update. J. Clin. Oncol. 32, 2255-2269 (2014).

2. Charehbili, A. et al. Neoadjuvant hormonal therapy for endocrine sensitive breast cancer: a systematic review. Cancer Treat. Rev. 40, 86-92 (2014).

3. Lumachi, F. et al. Endocrine therapy of breast cancer. Curr. Med. Chem. 18, 513-522 (2011).

4. Osborne, C. K. \& Schiff, R. Mechanisms of endocrine resistance in breast cancer. Annu. Rev. Med. 62, 233-247 (2011).

5. Normanno, N. et al. Mechanisms of endocrine resistance and novel therapeutic strategies in breast cancer. Endocr. Relat. Cancer 12, 721-747 (2005).

6. Dees, E. C. \& Carey, L. A. Improving endocrine therapy for breast cancer: it's not that simple. J. Clin. Oncol. 31, 171-173 (2013).

7. Arpino, G., Wiechmann, L., Osborne, C. K. \& Schiff, R. Crosstalk between the estrogen receptor and the HER tyrosine kinase receptor family: molecular mechanism and clinical implications for endocrine therapy resistance. Endocr. Rev. 29, 217-233 (2008).

8. Musgrove, E. A. \& Sutherland, R. L. Biological determinants of endocrine resistance in breast cancer. Nat. Rev. Cancer 9, 631-643 (2009).

9. Renoir, J. M., Bouclier, C., Seguin, A., Marsaud, V. \& Sola, B. Antioestrogenmediated cell cycle arrest and apoptosis induction in breast cancer and multiple myeloma cells. J. Mol. Endocrinol. 40, 101-112 (2008).

10. Conzen, S. D. Minireview: nuclear receptors and breast cancer. Mol. Endocrinol. 22, 2215-2228 (2008).

11. Naughton, C. et al. Progressive loss of estrogen receptor alpha cofactor recruitment in endocrine resistance. Mol. Endocrinol. 21, 2615-2626 (2007).

12. Zhang, Q. X., Borg, A., Wolf, D. M., Oesterreich, S. \& Fuqua, S. A. An estrogen receptor mutant with strong hormone-independent activity from a metastatic breast cancer. Cancer Res. 57, 1244-1249 (1997).

13. Toy, W. et al. ESR1 ligand-binding domain mutations in hormone-resistant breast cancer. Nat. Genet. 45, 1439-1445 (2013).

14. Robinson, D. R. et al. Activating ESR1 mutations in hormone-resistant metastatic breast cancer. Nat. Genet. 45, 1446-1451 (2013).
15. Merenbakh-Lamin, K. et al. D538G mutation in estrogen receptor-alpha: A novel mechanism for acquired endocrine resistance in breast cancer. Cancer Res. 73, 6856-6864 (2013).

16. Jeselsohn, R. et al. Emergence of constitutively active estrogen receptor-alpha mutations in pretreated advanced estrogen receptor-positive breast cancer. Clin. Cancer Res. 20, 1757-1767 (2014).

17. Clarke, R., Tyson, J. J. \& Dixon, J. M. Endocrine resistance in breast cancer-An overview and update. Mol. Cell. Endocrinol. 418(Pt 3): 220-234 (2015).

18. Beroukhim, R. et al. The landscape of somatic copy-number alteration across human cancers. Nature 463, 899-905 (2010).

19. Zack, T. I. et al. Pan-cancer patterns of somatic copy number alteration. Nat. Genet. 45, 1134-1140 (2013).

20. Cope, G. A. et al. Role of predicted metalloprotease motif of Jab1/Csn5 in cleavage of Nedd8 from Cul1. Science 298, 608-611 (2002).

21. Peth, A., Berndt, C., Henke, W. \& Dubiel, W. Downregulation of COP9 signalosome subunits differentially affects the CSN complex and target protein stability. BMC Biochem. 8, 27 (2007).

22. Cope, G. A. \& Deshaies, R. J. COP9 signalosome: a multifunctional regulator of SCF and other cullin-based ubiquitin ligases. Cell 114, 663-671 (2003).

23. Wei, N. \& Deng, X. W. The COP9 signalosome. Annu. Rev. Cell Dev. Biol. 19, 261-286 (2003).

24. Callige, M., Kieffer, I. \& Richard-Foy, H. CSN5/Jab1 is involved in liganddependent degradation of estrogen receptor $\{$ alpha\} by the proteasome. Mol. Cell. Biol. 25, 4349-4358 (2005).

25. Lavinsky, R. M. et al. Diverse signaling pathways modulate nuclear receptor recruitment of N-CoR and SMRT complexes. Proc. Natl Acad. Sci. USA 95, 2920-2925 (1998).

26. Jepsen, K. et al. Combinatorial roles of the nuclear receptor corepressor in transcription and development. Cell 102, 753-763 (2000).

27. Keeton, E. K. \& Brown, M. Cell cycle progression stimulated by tamoxifenbound estrogen receptor-alpha and promoter-specific effects in breast cancer cells deficient in N-CoR and SMRT. Mol. Endocrinol. 19, 1543-1554 (2005).

28. Shang, Y., Hu, X., DiRenzo, J., Lazar, M. A. \& Brown, M. Cofactor dynamics and sufficiency in estrogen receptor-regulated transcription. Cell 103, 843-852 (2000).

29. Metivier, R. et al. Estrogen receptor-alpha directs ordered, cyclical, and combinatorial recruitment of cofactors on a natural target promoter. Cell 115, 751-763 (2003).

30. Garraway, L. A. \& Janne, P. A. Circumventing cancer drug resistance in the era of personalized medicine. Cancer Discov. 2, 214-226 (2012).

31. Holohan, C., Van Schaeybroeck, S., Longley, D. B. \& Johnston, P. G. Cancer drug resistance: an evolving paradigm. Nat. Rev. Cancer 13, 714-726 (2013).

32. Stephens, P. J. et al. The landscape of cancer genes and mutational processes in breast cancer. Nature 486, 400-404 (2012).

33. Ciriello, G. et al. The molecular diversity of Luminal A breast tumours. Breast Cancer Res. Treat. 141, 409-420 (2013).

34. Girault, I. et al. Expression analysis of estrogen receptor alpha coregulators in breast carcinoma: evidence that NCOR1 expression is predictive of the response to tamoxifen. Clin. Cancer Res. 9, 1259-1266 (2003).

35. Wolf, D. A., Zhou, C. \& Wee, S. The COP9 signalosome: an assembly and maintenance platform for cullin ubiquitin ligases? Nat. Cell Biol. 5, 1029-1033 (2003).

36. Shackleford, T. J. \& Claret, F. X. JAB1/CSN5: a new player in cell cycle control and cancer. Cell Div. 5, 26 (2010).

37. Lee, M. H., Zhao, R., Phan, L. \& Yeung, S. C. Roles of COP9 signalosome in cancer. Cell Cycle 10, 3057-3066 (2011).

38. Pan, Y., Yang, H. \& Claret, F. X. Emerging roles of Jab1/CSN5 in DNA damage response, DNA repair, and cancer. Cancer Biol. Ther. 15, 256-262 (2014).

39. Kouvaraki, M. A. et al. Jun activation domain-binding protein 1 expression in breast cancer inversely correlates with the cell cycle inhibitor p27(Kip1). Cancer Res. 63, 2977-2981 (2003).

40. Wang, J. et al. Jab1 is a target of EGFR signaling in ERalpha-negative breast cancer. Breast Cancer Res. 10, R51 (2008).

41. Hsu, M. C. et al. Jabl is overexpressed in human breast cancer and is a downstream target for HER-2/neu. Mod. Pathol. 21, 609-616 (2008).

42. Adler, A. S. et al. CSN5 isopeptidase activity links COP9 signalosome activation to breast cancer progression. Cancer Res. 68, 506-515 (2008).

43. Lingaraju, G. M. et al. Crystal structure of the human COP9 signalosome. Nature 512, 161-165 (2014).

44. Soucy, T. A. et al. An inhibitor of NEDD8-activating enzyme as a new approach to treat cancer. Nature 458, 732-736 (2009).

45. Uhle, S. et al. Protein kinase CK2 and protein kinase D are associated with the COP9 signalosome. EMBO J. 22, 1302-1312 (2003).

46. Meerbrey, K. L. et al. The pINDUCER lentiviral toolkit for inducible RNA interference in vitro and in vivo. Proc. Natl Acad. Sci. USA 108, 3665-3670 (2011). 
47. Wiederschain, D. et al. Single-vector inducible lentiviral RNAi system for oncology target validation. Cell Cycle 8, 498-504 (2009).

48. Ma, Y. et al. Histone deacetylase 3 inhibits new tumour suppressor gene DTWD1 in gastric cancer. Am. J. Cancer Res. 5, 663-673 (2015).

49. Franken, N. A., Rodermond, H. M., Stap, J., Haveman, J. \& van Bree, C. Clonogenic assay of cells in vitro. Nat. Protoc. 1, 2315-2319 (2006).

50. Lu, R., Sun, M., Feng, J., Gao, X. \& Guo, L. Myofibrillogenesis regulator 1 (MR-1) is a novel biomarker and potential therapeutic target for human ovarian cancer. BMC Cancer 11, 270 (2011).

51. Lu, R. et al. Lactate dehydrogenase 5 expression in Non-Hodgkin lymphoma is associated with the induced hypoxia regulated protein and poor prognosis. PLoS ONE 8, e74853 (2013).

\section{Acknowledgements}

We are grateful to Dr J.G. Zhou for technical assistance and Dr J. Clark for critical review of the manuscript. We also acknowledge the scientific discussion with Drs J.G. Zhou and G. Wu. This work is supported by Science and Technology Commission of Shanghai Municipality (124119a0202), Shanghai Hospital Development Center (SHDC22014002) and Shanghai Pujiang Talent Plan Sponsorship (14PJD010). The project was also supported by the National Natural Science Foundation of China (NSF-81572552) and (81371178), and the Jiaxin municipal government to Gerchi Biotech Research Laboratories.

\section{Author contributions}

R.L., H.J., L.G. and P.Z. were responsible for the concept and experimental design. R.L., X.H., X.G., J.Z., X.X. and H.Z. performed the experiments. A.Z.Z., X.W. and H.J.

performed the data analyses. X.G. provided clinical samples and contributed to sample preparation. J.S. reviewed the patients' medical records and pathology. R.L., X.H., X.G. and P.Z. conceived the experiments, interpreted the data and wrote the manuscript. P.Z., R.L. and L.G. supervised the study.

\section{Additional information}

Supplementary Information accompanies this paper at http://www.nature.com/ naturecommunications

Competing financial interests: The authors declare no competing financial interests.

Reprints and permission information is available online at http://npg.nature.com/ reprintsandpermissions/

How to cite this article: Lu, R. et al. COPS5 amplification and overexpression confers tamoxifen-resistance in ER $\alpha$-positive breast cancer by degradation of NCoR. Nat. Commun. 7:12044 doi: 10.1038/ncomms12044 (2016).

(c) (i) This work is licensed under a Creative Commons Attribution 4.0 International License. The images or other third party material in this article are included in the article's Creative Commons license, unless indicated otherwise in the credit line; if the material is not included under the Creative Commons license, users will need to obtain permission from the license holder to reproduce the material. To view a copy of this license, visit http://creativecommons.org/licenses/by/4.0/

(C) The Author(s) 2016 